\title{
Ethnomedicinal knowledge of plants used as mono, di and polyherbal formulation for the treatment of common ailments and COVID-19 in the villages of Hamedan, Iran
}

\author{
Yusuf KAYA ${ }^{1 \otimes \square}$, Zohreh YOUSEFI ${ }^{1}$, Mohammad AKRAMI ${ }^{2}$ and Mahshad YOUSEFI ${ }^{3}$ \\ ${ }^{1} \mathrm{PhD}$, Department of Biology, Faculty of Sciences, Ataturk University, P.O.Box 25010, Erzurum, Turkey \\ ${ }^{2} \mathrm{PhD}$, Department of Agriculture, Payam e Noor University, P.O.Box 19395-4697, Tehran, Iran \\ ${ }^{3} \mathrm{MD}$, Hamadan University of Medical Sciences, Hamadan, Iran \\ Corresponding author's Email: ykaya@atauni.edu.tr; Web: https://avesis.atauni.edu.tr/ykaya; (DORCID: 0000-0003-3702-1895
}

\section{ABSTRACT}

Aim. This study aimed to document the native ethnomedicinal plants in mono, di and polyherbal combination used by people to cure various ailments, Qorveh-e Darjazin district of Hamedan province of Iran. Methods. Data were collected through both individual interviews and focus group discussions (FGDs) using interviewing 87 people in 28 villages. Plants voucher samples were also collected and classified with their families, botanical terms, local names, usages and miscellaneous matters via international and standard questionnaires. A total of 45 plant species belonging to 26 families are reported to be used for the treatment of a wide range of complaints, such as respiratory diseases like coronavirus (COVID-19), cardiovascular diseases, hypertension, ulcers, anemia, catarrh, digestive and urinary systems disorders, irregular menstruation, skin disorders, bone and rheumatic disorders etc. by monoherbal (45), diherbal (70) and polyherbal (57) ethnomedicinal formulations. Data were compared with those formerly gathered. Results. The authors found evidence that di and polyherbal known as Ayurvedic medicine is a preferred folk medicinal system of this area to prevent unwanted suffering of intense and prevalent ailments especially for irregular menstruation, anemia, calming the nervous systems, diarrhea, gastric ulcers, blood purification, hypertension, bone and rheumatic disorders, and severe acute respiratory syndrome coronavirus 2 (SARS-CoV- 2 or COVID-19) with the mixed formulation for natural elements in a particular ratio. Conclusion. The study reveals the rich polyherbal knowledge of the people of 28 villages of Hamadan that possess in traditional medicine. Recommendation. Polyherbal formulation of plants used by people of this area can be considered as an applicable safe strategy in control measures against novel diseases like COVID-19 and achieve greater therapeutic efficacy.

\begin{tabular}{l} 
Original Article \\
PII: S225199392100006-11 \\
Rec. 17 January 2021 \\
Rev. 19 May 2021 \\
Acc. 25 May 2021 \\
\hline
\end{tabular}

\section{Keywords}

Ailment,

Antioxidant, COVID-19,

Hamedan, Hyper inflammation, Nutraceuticals, Phytochemicals, Polyherbal formulation, Questionnaires, Traditional medicine

\section{INTRODUCTION}

Plants have been the basis of traditional medicine systems for thousands of years while they still widely practiced today to provide mankind with new remedies to attempt cures for diseases and to relive physical suffering [1]. So, the contributions of nature of our universe as a source of chemotherapeutic agents has always been continuing; so that, the main components of the drugs in clinical use are formed of natural products and their derivatives [2]. Chemical drugs are currently being replaced by herbal remedies especially in Asian countries [3] because of harmful, irreversible and side effects of their chemical structure on people [4, 5]. Pharmaceutical experts believe that of traditional medicine in developing countries is a main basis for maintaining good health $[3,6]$. Also, medicinal plants received much attention in agro-alimentary food, commerce, textile and allied industries [7], pharmaceutical and perfumes industries, and natural cosmetic products [8].

According to the World Health Organization's report, much of the world's population (about 80 percent) still rely on the traditional medicine or plant-derived medicines for their daily wellness and health needs [1]. It is estimated that a 80\% of Iran's population depend on plants to cure themselves [9]. In countries as the United States, about $60 \%$ of its population use plants to fight certain diseases and ailments. In India, Pakistan and China there is more demand of medicinal plants than of "official" medicines to cure themselves (80\%, 70\% and 40\%, respectively) [10]. Medicinal plants are extensively used in Indian traditional medicine called Apurveda [11, 12]. 
Although the use of plants in phytochemical medicine despite the availability of medicinal plants has decreased but substantial information about folk medicine still remain among the population [6, 13, 14]. People who use folk herbal remedies may not understand the scientific rationale behind that, and they just know from personal experience based on using therapeutic doses of some highly usable medicinal plants. Hence, recording information and focusing on the ethno pharmacy researches of small districts permits the rediscovery of the new traces of retained traditional knowledge.

Iran's flora comprises about 6417 species, 611 subespecie, 465 variedad and 83 híbrido, 1810 taxon of which are endemic [15]. The first written versions derived from medicinal plants, goes back to $3000 \mathrm{BC}$ including the world's major civilizations of Persia, Egypt, Middle East, Ancient Greece, India and China [16]. Iranian people have utilized plants as medicinal remedies, as well as for food, dyes, fuel, ornament, furniture, agricultural tools, construction materials and cosmetics.

The Qorveh-e Darjazin district of Hamedan is consisted of 28 villages and a population of 40299 people in year 2006 [17]. Most of the population that is engaged in subsistence agriculture has also been found professional in using medicinal plants to treat a wide range of ailments and diseases. Therefore, in line with the needs of people of the global majority to medicinal plants and drug discovery, an ethnobotanical survey was made of Qorveh-e Darjazin district of Hamedan province, Iran. The main purpose of the study was to identify medicinal usage of the herbs individually or in polyherbal formulation used by people of this area to cure various ailments especially the coronavirus (COVID-19) global pandemic.

\section{MATERIALS AND METHODS}

\section{Study area}

Hamedan province is located in the West of Iran, and has 28 cities (Figure 1) [18]. Hamadan is known as city of Avicenna (Ibn Sina) who died in June 1037, in his fifty-sixth year and was buried in Hamadan, Iran [19]. He was one of the most celebrated physicians in the early Islamic Empire and he wrote medical texts in a wide range of subjects. The most significant books in the history of medicine are the Book of Healing (the Kitab alShifa) [19] and the Canon of Medicine (Al-Qanun fi't-Tibb) [20]. Both book remained a major authority for medical students in both the Islamic world and Europe until well into the 1700 s.

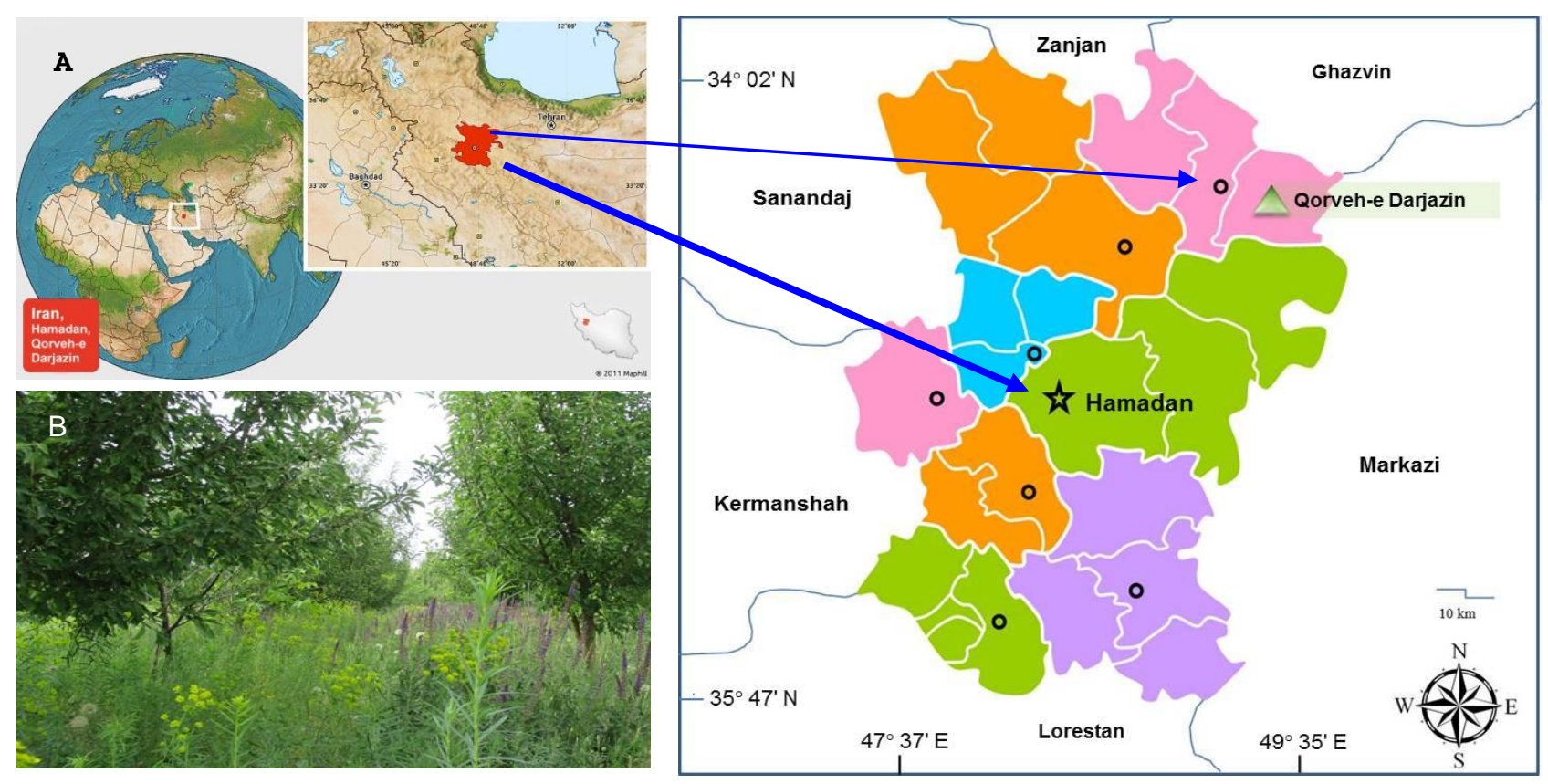

Figure 1. A) Investigation site: Qorveh-e Darjazin district (ム); B) A selected field of Soozan village, Qorveh-e Darjazin district. This district have two main villages (named Nir and Changarin) and 26 oasis villages such as Qara Qāyeh, Nezam Abad, Karafs, Vasmaq, Shavand, Savar, Darjazin, Sonqorabad, Alborz, Piri Beyk, Valashjrd, Razin, Iman, Ahmadabad, Pelican, Wrekin, Poshtejin, Sherkat, Navar, Soozan, Kaaj, Kamandan, Kahard, Behkandan etc.

The Qorveh-e Darjazin district is located North East of Hamedan, situated between $35^{\circ} 621^{\prime} \mathrm{N}$ and $49^{\circ} 10^{\prime} \mathrm{E}$. Its area is $759 \mathrm{~km}^{2}$. The district is $85 \mathrm{~km}$ away from the provincial capital and is part of Razan town. It is located 
in the area known as Darjazin that have a very ancient history and famous people of this region have raised. According to the census of Iran, the population of Qorveh-e Darjazin district in 2006 was about 40299 people [17].

This district has two main villages (named Nir and Changarin) and 26 oasis villages such as Darjazin, Soozan, Shavand, etc. (Figure 1). The people of Qorveh-e Darjazin district and all its subsidiaries villages speak Azerbaijani known as Azeri-Turkish language. Most of the resident population in this area is engaged in subsistence agriculture and products such as wheat, barley, grapes are widely cultivated. The soil is made up primarily of types of limestone and most of the land consists of quaternary alluvial deposits. The altitude ranges from 1735 to $1850 \mathrm{~m}$, and the dominant vegetation is pasture and crop plants with durability (viability) and resistant to cold. The coupon method climate of the region is cold semi-arid, cold and snowy in winter and temperate in the summer. The annual mean temperature value is $5.11{ }^{\circ} \mathrm{C}$ ( $\mathrm{Min}$ is $-34^{\circ} \mathrm{C}$ and $\mathrm{Max}$ is $24{ }^{\circ} \mathrm{C}$ ). The area is covered with snow from November to April $[17,21]$.

\section{Data collection}

The study started from December 2013 and information related to the ethnobotanical practices were collected during December 2015-July 2016 from different areas of Qorveh-e Darjazin district. All 28 villages were screened to collect ethnobotanical information using semi-structured and structured questionnaires according to Özgen et al. [6] including identification data such as local name, part used, and medicinal usage for plant. Interviews were held with 87 people ( 47 women, 40 men) and most of the interviewees were over 40 years old and belonged to those that still are traditional farmers with pastoral activities, mainly sheep or goat breeding. All the information about plant species were collected and then identified. Samples of used standard questionnaires are listed on a page at the end of the paper.

For the interviews, people who were locally expert in medicinal plants were asked to describe the method of preparation and use of the medical remedies for each plant species. Meanwhile, some fresh and dried plant specimens were shown to the participated informants. Some of the collected plant specimens were dried and taxonomically identified by botanists by using floristic and taxonomic references, especially Flora Iranica to confirm the plant names [22] and some of the specimens were collected for only identification and were not deposited in herbaria. The scientific names of species were checked and validated taxonomically via the plant

list (www.theplantlist.org) and the international plant names index (http://www.ipni.org/ipni/plantnamesearchpage.do). In addition, the ethno medicinal data were analyzed according to plant families, traditional preparation, and part used.

\section{Preference ranking for formulations (PRFs)}

A total of 45 plant species belonging to 26 families were used in 45 monoherbal, 70 diherbal (two plants) and 57 polyherbal ethnomedicinal formulations for curing particular ailments (jaundice, wind, bone and rheumatic disorders, hypertension, arrhythmia, calming the nervous systems, irregular menstruation, diarrhea, eye reinforcement, stomach aches, gastric ulcers, and heartburn, anemia, lipid and cholesterol lowering, blood purification, cold and fever, respiratory diseases, increasing intelligence quotient (IQ), the sexual power and sperm count and motility, sunstroke, COVID-19, skin disorders, urinary system disorders, and digestive system diseases). Informants selected for preference ranking were asked to present the values ( $A=$ best, $\mathrm{B}=$ very good, $\mathrm{C}=$ good, $\mathrm{D}=$ less used, $\mathrm{E}=$ don't know) to the different herbal formulations plus modes of preparation only for di and polyherbal formulations. The percentage of voters which agreed with each value were calculated up and placed for various ailments. The most used plants in different formulations for different ailments and their number of times has also been presented.

\section{RESULTS}

\section{Ethnomedicinal plants, modes of preparation and utilization pattern}

A total of 45 specimens belonging to 26 plant families were recorded as folk medicines being used in the villages of Qorveh-e Darjazin district. Based on the 45 specimens, 76 medicinal plant usages were explained for 55 herbal folk remedies. Table 1 shows the obtained results and lists plants used as remedies in alphabetical order by family as well as the most important medicinal plant usages.

The families with the highest number of reported medicinal species were Asteraceae (5 specimens), Lamiaceae (4 specimens), Apiaceae (4 specimens), Fabaceae (3 specimens). For each species, the following 
ethnobotanical and pharmacognostic information are provided: scientific name and number, local or vernacular name, preparations, parts used, therapeutic uses, citations, and percentage of reports, which was 401 . The 45 medicinal plant species including the wild and none-wild were collected from cultivated material (Table 1). The species with the highest uses' records as special herbal medicine in the Qorveh-e Darjazin area were Achillea eriophora DC., Cichorium intybus L., Althea officinalis L., Borago officinalis L., Mentha spp., Salix aegyptiaca L., Melissa officinalis L., Thymus kotschyanus Boiss. \& Hohen. (Table 1).

The most common traditional or local forms of preparation and application of the 49 herbal remedies from 45 medicinal plant species were decoction (as tea), eaten fresh, powder and distillate, respectively (Tables 1-3, Figure 2). Results also showed that people in this region usually use seeds that have better possibility of polyherbal combination with the reported herbs, fruits, leaves and flowers in the different mixtures, formulation and preparation forms, to achieve greater therapeutic usage and reduce the toxicity, without any side effects upon the interviewer's claim (Tables 3 and 4, Figure 3).

Table 4 showed therapeutic uses of the 49 herbal remedies from 45 medicinal plant species and Polyherbal combination possibility of medicinal plants species for the treatment of prevalent diseases. In this table, the disease states treated (expressed as percentage of the total records) are summarized. The local people choose herbal remedies mainly for treat digestive system diseases (11.8\%), urinary system disorders (7.89\%), skin disorders and rash, hemorrhoids and sunstroke (6.57\%), flu and lung diseases, cold and fever, blood purification and or improving sexual stamina and boosting semen volume, sperm count and motility (5.26\%), for lipid and cholesterol lowering, treatment of anemia, stomach aches, gastric ulcers, heartburn and enhancing intelligence (3.94\%), bone and rheumatic disorders, hypertension, biliary excretion, arrhythmia, calming the nervous system, menstrual irregularities, diarrhea, eye movements and reinforcement and currently for treatment of severe respiratory diseases like COVID-19.

Results of table 4 showed that for treatment of ailments, the medicinal plant species are used in mono, di and polyherbal combination by people of these area's villages. According to the results, only one species (Avena sativa L.) is used for jaundice treatment and also Carum carvi L. is used as monoherbal plant for wind treatment.

The following plants are used in mono and diherbal combination: Brassica oleracea L. and Phaseolus mungo L. are used for treatment of bone diseases and rheumatism; Allium sativum L. and Morus nigra L. are used against hypertension; Mentha spp. and Borago officinalis L. for arrhythmia; Melissa officinalis L. and Salix aegyptiaca L. for calming the nervous system; Thymus kotschyanus Boiss. \& Hohen. and Achillea eriophora DC. against menstrual irregularities in women; Thymus kotschyanus Boiss. \& Hohen. and Mentha spp. against diarrhea; Centaurium cyanus L. and Calendula officinalis L. for control of biliary excretion; Geranium spp. and Origanium diktamnus L. for eye movements and reinforcement; Amygdalus communis L. and Corylus avellana L. for cosmetics; and Rhus coriaria L., Citrus bergamia Risso and Cichorium intybus L. for decrease lipid and cholesterol.

The following plants are used in mono, di and polyherbal combination: Spinacia oleraceael L. and Cicer arietinum L., Phaseolus mungo L. against anemia; Berberis integerrima L., Citrus bergamia Risso, Punica granatum L. and Borago officinalis L. for blood purification; Muscari comosum (L.) Mill. and Amygdalus communis L., Juglans regia L., Corylus avellana L. for enhancing intelligence quotient (IQ), the sexual power, sperm count and motility, and for cosmetics uses; Althea officinalis L., Beta vulgaris L., Malva splvestris L., Brassica rapa L., Origanum dictamnus L., Salix aegyptiaca L., Cichorium intybus L., Thymus kotschyanus Boiss. \& Hohen. and Mentha spp. are used for ailments like cold and fever, flu and lung diseases and severe acute respiratory syndrome coronavirus 2 (SARS-CoV-2 or COVID-19); Salix aegyptiaca L., Cichorium intybus L., Thymus kotschyanus Boiss. \& Hohen., Mentha spp. and Origanum dictamnus L. against sunstroke; Achillea eriophora DC., Aethusa cynapium L., Nigella sativa L., Chenopodium spp., Berberis integerrima Bunge and Plantago major L. are used for skin disorders and rash; Borago officinalis L., Citrulus vulgaris L., Zea mays L., Apium graveolens L., Thymus kotschyanus Boiss. \& Hohen. and Tragopogoh collinus L. are used for urinary system disorders; and Anemone coronaria L., Astragalus adscendens Boiss \& Hausskn., Centaurium cyanus L., Calendula officinalis L., Cucumis sativus L., Cyperus officinalis L., Ficus carica L., Tragopogon collinus DC., Thymus kotschyanus Boiss. \& Hohen., Plantago major L., are used for digestive system ailments.

Many of the villages' people stated that a combination of the related medicinal plants can be so effective when the ailment is intense and serious and they believe it can be considered as an applicable strategy in control measures against diseases and improve primary health care. Medicinal plants typically contain mixtures of phytochemical compounds that may act in synergy, additively or individually to improve health [12, 23, 24]. Since no disease has just one single symptom and many different factors may interfere in the 
pathogenesis of a disease, there is a need to different medicinal plants to resolve the various signs and symptoms of a disease $[25,26]$. The various plants in a polyherbal medicine may increase the affectivity and potency of the formulation, reduce unwanted effects, make the formulation more palatable and increase its lifespan. In fact, all the herbs in a polyherbal formulation are active and have their own effect [27]. The polyherbalism is known as an ethnomedicinal formulation with a better therapeutic effect and lower toxicity to help patients easily restore their balance for a long healthy life [13].

This study's data and observations obtained from village people were compared with information formerly recorded from other regions of Iran [28-33] as well as earlier data from the neighbor area in Hamedan city [3, 34, 35]. This is likely one of the first reports of some plant species being used in folk medicine in Qorveh-e Darjazin district of Hamedan, and unique research in Iran. The species concerned are Achillea eriophora DC., Aethusa cynapium V, Allium sativum V, Althea officinalis L., Amygdalus communis L., Anemone coronaria L., Apium graveolens L., Astragalus adscendens Boiss \& Hausskn., Avena sativa L., Berberis integerrima L., Beta vulgaris L., Borago officinalis L., Brassica oleracea L., Brassica rapa L., Calendula officinalis L., Carum carvi L., Centaurium cyanus L., Chenopodium spp., Cichorium intybus L., Citrullus vulgaris L., Citrus bergamia Risso, Conium maculatum L., Corylus avellana L., Cucumis sativus L., CYperus officinalis L., Ficus carica L., Geranium spp., Juglans regia L., Melissa officinalis L., Mentha spp., Malva splvestris L., Marus nigra L., Muscari comosum (L.) Mill., Nigella sativa L., Origanum dictamnus L., Cicer arietinum L., Phaseolus mungo L., Punica granatum L., Rhus coriaria L., Spinacia oleracea L., Salix aegyptiaca L., Plantago major L., Thymus lancifolius Celak L., Tragopogon collinus DC., and Zea mays L.

Table 1. Medicinal plants used in the villages of Qorveh-e Darjazin district, Hamedan, Iran

\begin{tabular}{|c|c|c|c|c|c|c|c|}
\hline \multirow{2}{*}{ No. } & \multirow{2}{*}{$\begin{array}{l}\text { Family and } \\
\text { botanical name } \\
\text { (A-Z) }\end{array}$} & \multirow{2}{*}{$\begin{array}{l}\text { Local names in Phonetics, } \\
\text { Persian, Local Azeri (AZ), } \\
\text { and Turkish (TR), } \\
\text { respectively }\end{array}$} & \multirow{2}{*}{$\begin{array}{l}\text { Parts } \\
\text { used }\end{array}$} & \multirow{2}{*}{ Uses/ailments treated } & \multirow{2}{*}{ Preparations } & \multicolumn{2}{|c|}{ Citations } \\
\hline & & & & & & (n) & (\%) \\
\hline \multicolumn{8}{|c|}{ Amaranthaceae } \\
\hline 1 & Chenopodium spp. & $\begin{array}{l}\text { /kæzja:kı/ } \\
\text { غاز ياقى } \\
\text { Ghazyaghi (AZ) } \\
\text { Kazayağı (AZ, TR) }\end{array}$ & Herb & Skin rash with itching & $\begin{array}{l}\text { Powder and } \\
\text { decoction }\end{array}$ & 3 & 0.74 \\
\hline 2 & Spinacia oleracea L. & $\begin{array}{l}\text { /osfina:ḋ/ } \\
\text { Spanakh (AZ) } \\
\text { Abdestbozanotu (TR) } \\
\end{array}$ & $\begin{array}{l}\text { Leaves } \\
\text { and } \\
\text { stem }\end{array}$ & Treatment of anemia & Fresh or cooking & 20 & 4.98 \\
\hline \multicolumn{8}{|c|}{ Amaryllidaceae } \\
\hline 3 & Allium sativum L. & $\begin{array}{l}\text { /si:r/ } \\
\text { سير } \\
\text { Sarımsak (AZ, TR) }\end{array}$ & Fruit & $\begin{array}{l}\text { High blood pressure } \\
\text { lowering }\end{array}$ & Eaten fresh & 15 & 3.74 \\
\hline \multicolumn{8}{|c|}{ Anacardiaceae } \\
\hline 4 & Rhus coriaria L. & $\begin{array}{l}\text { /so:ma:k/ } \\
\text { سماق } \\
\text { Sumak (AZ, TR) }\end{array}$ & Fruit & $\begin{array}{l}\text { Lipid and cholesterol } \\
\text { lowering }\end{array}$ & Powder eaten & 8 & 1.99 \\
\hline \multicolumn{8}{|c|}{ Apiaceae } \\
\hline 5 & Aethusa cynapium L. & $\begin{array}{l}\text { / أشوكران صغير } \\
\text { Shokaran-e-Sagir (AZ) } \\
\text { Köpek, Maydanozu (TR) }\end{array}$ & Herb & $\begin{array}{l}\text { Animal bites and Snake } \\
\text { bites }\end{array}$ & Decoction & 1 & 0.24 \\
\hline 6 & Apium graveolens L. & $\begin{array}{l}\text { /kæræfs/ } \\
\text { Sرفf } \\
\text { Karafs (AZ) } \\
\text { Kereviz (TR) } \\
\end{array}$ & Herb & $\begin{array}{l}\text { Dissolve kidney stones or } \\
\text { renal calculi }\end{array}$ & Decoction & 9 & 2.24 \\
\hline 7 & Carum carvi L. & $\begin{array}{l}\text { /zi:re sija:h/ } \\
\text { زيرهى سياه } \\
\text { Kara Zire (AZ) } \\
\text { Şifali Kimyon (TR) }\end{array}$ & Seed & Trapped gas or wind pain & Decoction & 2 & 0.49 \\
\hline 8 & $\begin{array}{l}\text { Conium maculatum } \\
\text { L. }\end{array}$ & $\begin{array}{l}\text { / شوكران } \\
\text { Shokaran (AZ) } \\
\text { Baldıran, Ağuotu, } \\
\text { Başdöndürenotu (TR) } \\
\end{array}$ & Herb & $\begin{array}{l}\text { Stomach aches, gastric } \\
\text { ulcers, and heartburn }\end{array}$ & $\begin{array}{l}\text { Crushed with } \\
\text { yogurt }\end{array}$ & 1 & 0.24 \\
\hline \multicolumn{8}{|c|}{ Asparagaceae } \\
\hline 9 & $\begin{array}{l}\text { Muscari comosum (L.) } \\
\text { Mill. }\end{array}$ & $\begin{array}{l}\text { /kæla:kæke ku:fəجI/ } \\
\text { كلاغكـ خوشاى } \\
\text { Chanakh Sindiren (Az) } \\
\text { Arap sümbülü (TR) } \\
\end{array}$ & Herb & $\begin{array}{l}\text { Improving sexual } \\
\text { stamina and boosting } \\
\text { semen volume, sperm } \\
\text { count and motility } \\
\end{array}$ & $\begin{array}{l}\text { Powder } \\
\text { (eaten as mixed } \\
\text { with honey) }\end{array}$ & 2 & 0.49 \\
\hline \multicolumn{8}{|c|}{ Asteraceae } \\
\hline 10 & $\begin{array}{l}\text { Achillea eriophora } \\
\text { DC. }\end{array}$ & $\begin{array}{l}\text { /bu;ma:dær } 1 \text { n/ } \\
\text { بومادران }\end{array}$ & Herb & $\begin{array}{l}\text { Itching and menstrual } \\
\text { irregularities }\end{array}$ & $\begin{array}{l}\text { Distillate, } \\
\text { decoction and }\end{array}$ & 9 & 2.24 \\
\hline
\end{tabular}




\begin{tabular}{|c|c|c|c|c|c|c|c|}
\hline & & Bumadaran (AZ, TR) & & & ointment & & \\
\hline 11 & $\begin{array}{l}\text { Calendula officinalis } \\
\text { L. }\end{array}$ & $\begin{array}{l}\text { /hæmi:fe bæha:r/ } \\
\text { هميشه بهار } \\
\text { HamisheBahar (AZ) } \\
\text { Aynisefa (TR) }\end{array}$ & Herb & Biliary excretion & Soaking in water & 1 & 0.24 \\
\hline 12 & Centaurium cyanus L. & $\begin{array}{l}\text { /gə:le gændə:m/ } \\
\text { كن كند } \\
\text { Ghol-e Ghandom (Az) } \\
\text { Maviçiçek (TR) }\end{array}$ & Flower & Biliary excretion & Decoction & 2 & 0.49 \\
\hline 13 & Cichorium intybus L. & $\begin{array}{l}\text { /ka:sni/ } \\
\text { Kasni (AZ) } \\
\text { Yabani Hindiba (TR) } \\
\end{array}$ & Herb & $\begin{array}{l}\text { Heat exhaustion, } \\
\text { sedative, blood purifier, } \\
\text { lipid and cholesterol } \\
\text { lowering }\end{array}$ & $\begin{array}{l}\text { Decoction and } \\
\text { distillate }\end{array}$ & 8 & 1.99 \\
\hline 14 & $\begin{array}{l}\text { Tragopogon collinus } \\
\text { DC. }\end{array}$ & $\begin{array}{l}\text { / شنى } 1 \text { / } \\
\text { Yemlik (AZ) } \\
\text { Sarı yemlik (TR) } \\
\end{array}$ & Leaves & $\begin{array}{l}\text { Bowel infections, } \\
\text { stomach aches, gastric } \\
\text { ulcers, and heartburn }\end{array}$ & $\begin{array}{l}\text { Fresh eaten or } \\
\text { cooking }\end{array}$ & 8 & 1.99 \\
\hline \multicolumn{8}{|c|}{ Berberidaceae } \\
\hline 15 & $\begin{array}{l}\text { Berberis integerrima } \\
\text { Bunge }\end{array}$ & $\begin{array}{l}\text { /zerefk/ } \\
\text { زرشك } \\
\text { Zereshk (AZ) } \\
\text { Sarıçalı Kızambık (TR) }\end{array}$ & Fruit & $\begin{array}{l}\text { Blood cleanser to cure } \\
\text { pimples and acne }\end{array}$ & Decoction & 11 & 2.74 \\
\hline \multicolumn{8}{|c|}{ Betulaceae } \\
\hline 16 & Corylus avellana L. & $\begin{array}{l}\text { /fændo:k/ } \\
\text { فندق } \\
\text { Findlk (AZ, TR) }\end{array}$ & Seed & $\begin{array}{l}\text { Increasing intelligence } \\
\text { quotient (IQ), the sexual } \\
\text { power, sperm count and } \\
\text { motility, and cosmetics } \\
\text { uses }\end{array}$ & $\begin{array}{l}\text { Eaten fresh, } \\
\text { powder eaten, and } \\
\text { burned }\end{array}$ & 11 & 2.74 \\
\hline \multicolumn{8}{|c|}{ Boraginaceae } \\
\hline 17 & Borago officinalis L. & $\begin{array}{l}\text { /go:le ga:v zæba:n/ } \\
\text { كل كاو زبان } \\
\text { Gholghavzaban (AZ) } \\
\text { Hodan (TR) }\end{array}$ & Flower & $\begin{array}{l}\text { Blood purifier, dissolve } \\
\text { kidney stones and heart } \\
\text { arrhythmia }\end{array}$ & Decoction & 11 & 2.74 \\
\hline \multicolumn{8}{|c|}{ Brassicaceae } \\
\hline 18 & Brassica oleracea L. & $\begin{array}{l}\text { /kælæm pi:t } \int / \\
\text { كل بيجج (AZ) } \\
\text { Kalam (TR) } \\
\text { Lahana (TR }\end{array}$ & Leaves & $\begin{array}{l}\text { Gout and bone loss } \\
\text { treatment and }\end{array}$ & Eat in fresh & 6 & 1.49 \\
\hline 19 & Brassica rapa L. & $\begin{array}{l}\text { / شلغم } \\
\text { Salgam (AZ, TR) }\end{array}$ & Root & Catarrh and chest pain & Decoction & 18 & 4.48 \\
\hline \multicolumn{8}{|c|}{ Chenopodiaceae } \\
\hline 20 & Beta vulgaris L. & 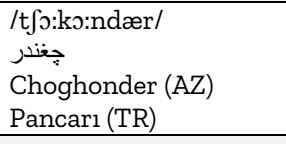 & Root & $\begin{array}{l}\text { Cold and fever and chest } \\
\text { pain }\end{array}$ & $\begin{array}{l}\text { Fresh and } \\
\text { Decoction }\end{array}$ & 13 & 3.24 \\
\hline \multicolumn{8}{|c|}{ Curcurbitaceae } \\
\hline 21 & $\begin{array}{l}\text { Citrullus vulgaris } \\
\text { Schrad. }\end{array}$ & $\begin{array}{l}\text { /hendeva:ne/ } \\
\text { هندو انذه } \\
\text { Karpuz (AZ, TR) }\end{array}$ & Fruit & Dissolve kidney stones & $\begin{array}{l}\text { Decoction and skin } \\
\text { of fruit }\end{array}$ & 5 & 1.24 \\
\hline 22 & Cucumis sativus L. & $\begin{array}{l}\text { /ki ja:r/ } \\
\text { خيار } \\
\text { Khiyar (AZ) } \\
\text { Hiyar (TR) } \\
\end{array}$ & Fruit & Constipation disposal & Eaten as fresh & 6 & 1.49 \\
\hline \multicolumn{8}{|c|}{ Cyperaceae } \\
\hline 23 & $\begin{array}{l}\text { Cyperus officinalis } \\
\text { T.Nees }\end{array}$ & $\begin{array}{l}\text { /ba:da:m zæmi:nI/ } \\
\text { بادام مينىى } \\
\text { Yer Bademi (AZ) } \\
\text { Yer Fistığ1 (TR) }\end{array}$ & Fruit & Constipation disposal & Eaten as fresh & 5 & 1.24 \\
\hline \multicolumn{8}{|c|}{ Fabaceae } \\
\hline 24 & $\begin{array}{l}\text { Astragalus } \\
\text { adscendens Boiss. \& } \\
\text { Hausskn. }\end{array}$ & $\begin{array}{l}\text { /gævæn/ } \\
\text { Gَ' } \\
\text { Geven (AZ) } \\
\text { Geven Out (TR) }\end{array}$ & Herb & $\begin{array}{l}\text { Swelling of the stomach } \\
\text { and intestines, gastric } \\
\text { ulcers }\end{array}$ & $\begin{array}{l}\text { Powder (eaten with } \\
\text { honey) }\end{array}$ & 3 & 0.74 \\
\hline 25 & Cicer arietinum L. & $\begin{array}{l}\text { /no:ko:d/ } \\
\text { نخود } \\
\text { Nokhut (AZ) } \\
\text { Nohut (TR) } \\
\end{array}$ & Seed & $\begin{array}{l}\text { Increasing blood iron } \\
\text { levels }\end{array}$ & Cooked & 14 & 3.49 \\
\hline 26 & Phaseolus mungo L. & $\begin{array}{l}\text { /ma:f/ } \\
\text { ماش } \\
\text { Mash (AZ) } \\
\text { Fasulye, Mung (TR) }\end{array}$ & Seed & $\begin{array}{l}\text { Increasing blood and } \\
\text { bone loss treatment }\end{array}$ & Fresh & 12 & 2.99 \\
\hline \multicolumn{8}{|c|}{ Geraniaceae } \\
\hline 27 & Geranium spp. & 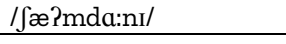 & Flower & Eye movements and & Decoction & 2 & 0.49 \\
\hline
\end{tabular}




\begin{tabular}{|c|c|c|c|c|c|c|c|}
\hline & & $\begin{array}{l}\text { شمدانى } \\
\text { Shamdani (AZ) } \\
\text { Sardunya (TR) }\end{array}$ & $\begin{array}{l}\& \\
\text { Leaves }\end{array}$ & reinforcement & & & \\
\hline \multicolumn{8}{|c|}{ Juglandaceae } \\
\hline 28 & Juglans regia L. & $\begin{array}{l}\text { /gerdu:/ } \\
\text { كردو } \\
\text { Goz (AZ) } \\
\text { Ceviz (TR) }\end{array}$ & Seed & $\begin{array}{l}\text { Improving sexual } \\
\text { stamina and boosting } \\
\text { semen volume, sperm } \\
\text { count and motility and } \\
\text { enhancing intelligence }\end{array}$ & Eaten fresh or Dry & 13 & 3.24 \\
\hline \multicolumn{8}{|c|}{ Lamiaceae } \\
\hline 29 & Melissa officinalis L. & $\begin{array}{l}\text { /ba:drændzbu:/ } \\
\text { بادرنجبو } \\
\text { Badresncbu (AZ) } \\
\text { Oğul out (TR) }\end{array}$ & Herb & $\begin{array}{l}\text { Calming the nervous } \\
\text { system and } \\
\text { schizophrenia }\end{array}$ & Distillate & 10 & 2.49 \\
\hline 30 & Mentha spp. & $\begin{array}{l}\text { /næ?na:?/ } \\
\text { نعناع } \\
\text { Nana (AZ) } \\
\text { Nane (TR) }\end{array}$ & Herb & $\begin{array}{l}\text { Cardiovascular diseases, } \\
\text { diarrhea, heat exhaustion } \\
\text { and Sedative }\end{array}$ & $\begin{array}{l}\text { Fresh, Decoction } \\
\text { and distillate }\end{array}$ & 16 & 3.99 \\
\hline 31 & $\begin{array}{l}\text { Origanum dictamnus } \\
\text { L. }\end{array}$ & $\begin{array}{l}\text { /pu:ne ku:hi/ } \\
\text { يونه كو } 1 \\
\text { Keklikotu (AZ) } \\
\text { Girit kekiği (TR) }\end{array}$ & Herb & $\begin{array}{l}\text { Lung disease, heat } \\
\text { exhaustion, sedative, eye } \\
\text { movements and } \\
\text { reinforcement }\end{array}$ & $\begin{array}{l}\text { Decoction and } \\
\text { distillate }\end{array}$ & 5 & 1.24 \\
\hline 32 & $\begin{array}{l}\text { Thymus kotschyanus } \\
\text { Boiss. \& Hohen. }\end{array}$ & 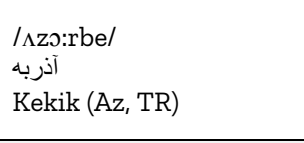 & $\begin{array}{l}\text { Leaves } \\
\& \\
\text { Branch }\end{array}$ & $\begin{array}{l}\text { Diarrhea, bowel } \\
\text { infections, menstrual } \\
\text { irregularities, heat } \\
\text { exhaustion and sedative }\end{array}$ & $\begin{array}{l}\text { Powder fresh, } \\
\text { decoction, distillate }\end{array}$ & 16 & 3.99 \\
\hline \multicolumn{8}{|c|}{ Lythraceae } \\
\hline 33 & Punica granatum L. & $\begin{array}{l}\text { /æna:r/ } \\
\text { انار } \\
\operatorname{Nar}(A Z, T R) \\
\end{array}$ & Seed & Blood purifier & Fresh & 16 & 3.99 \\
\hline \multicolumn{8}{|c|}{ Malvaceae } \\
\hline 34 & Althea officinalis L. & $\begin{array}{l}\text { /kætmi/ } \\
\text { ختمى } \\
\text { Khetmi (AZ) } \\
\text { Tibbi Hatmi (TR) }\end{array}$ & $\begin{array}{l}\text { Flower } \\
\text { \& seed }\end{array}$ & Cold and fever treatment & Decoction & 14 & 3.49 \\
\hline 35 & Malva sylvestris L. & $\begin{array}{l}\text { /pæni:ræk/ } \\
\text { Fنيرك } \\
\text { Fetirek (AZ) } \\
\text { Yabani ebegümeci (TR) }\end{array}$ & Herb & $\begin{array}{l}\text { Laxative and fever } \\
\text { treatment }\end{array}$ & Decoction & 2 & 0.49 \\
\hline \multicolumn{8}{|c|}{ Moraceae } \\
\hline 36 & Ficus carica L. & $\begin{array}{l}\text { /ændzi:r/ } \\
\text { انجير } \\
\text { Encir (AZ) } \\
\text { Inciri (TR) }\end{array}$ & Fruit & $\begin{array}{l}\text { Chronic constipation } \\
\text { disposal }\end{array}$ & Fresh and dry & 17 & 4.23 \\
\hline 37 & Morus nigra L. & $\begin{array}{l}\text { / شa:tu:t/ } \\
\text { شاه توت } \\
\text { Shahtut (AZ) } \\
\text { Dut, Karadut (TR) }\end{array}$ & Fruit & $\begin{array}{l}\text { High blood pressure } \\
\text { lowering }\end{array}$ & Fresh eaten & 21 & 5.23 \\
\hline \multicolumn{8}{|c|}{ Plantaginaceae } \\
\hline 38 & Plantago major L. & $\begin{array}{l}\text { /ba:rhæng/ } \\
\text { بارهن } \\
\text { Baharang (AZ) } \\
\text { Büyük Sinirliot (TR) }\end{array}$ & Seed & $\begin{array}{l}\text { Acne and skin softening } \\
\text { and dispose of excess } \\
\text { fluids, Gastrointestinal } \\
\text { bleeding }\end{array}$ & Decoction & 3 & 0.74 \\
\hline \multicolumn{8}{|c|}{ Poaceae } \\
\hline 39 & Avena sativa L. & $\begin{array}{l}\text { /dzo:je do: sær/ } \\
\text { جو دوسر } \\
\text { Arpa (AZ) } \\
\text { Yulaf (TR) }\end{array}$ & Seed & Jaundice treatment & Decoction & 4 & 0.99 \\
\hline 40 & Zea mays L. & $\begin{array}{l}\text { /bæla:l/ } \\
\text { Zorret (AZ) } \\
\text { Misir (TR) } \\
\end{array}$ & $\begin{array}{l}\text { Flower } \\
\text { (Tassel) }\end{array}$ & Strong diuretic & Decoction & 3 & 0.74 \\
\hline \multicolumn{8}{|c|}{ Ranunculaceae } \\
\hline 41 & Anemone coronaria L. & $\begin{array}{l}\text { /la:le so:rk/ } \\
\text { لاله سرخ } \\
\text { Kirmizi lale (AZ) } \\
\text { Taçlı dağ lalesi (TR) }\end{array}$ & Flower & Mouth ulcers & Decoction & 1 & 0.24 \\
\hline 42 & Nigella sativa L. & $\begin{array}{l}\text { /sıja:h da:ne/ } \\
\text { سياه دانه } \\
\text { Gharaca (Az) } \\
\text { Çörek، Çörekotu (TR) }\end{array}$ & Herb & Removing warts & $\begin{array}{l}\text { Powder, white } \\
\text { vinegar, ointment }\end{array}$ & 1 & 0.24 \\
\hline
\end{tabular}




\begin{tabular}{|c|c|c|c|c|c|c|c|}
\hline \multicolumn{8}{|c|}{ Rosaceae } \\
\hline 43 & $\begin{array}{l}\text { Amygdalus } \\
\text { communis L. }\end{array}$ & $\begin{array}{l}\text { /ba:da:m/ } \\
\text { Badam (AZ) } \\
\text { Badem (TR) }\end{array}$ & Seed & $\begin{array}{l}\text { Boosting sperm count } \\
\text { and motility, IQ, } \\
\text { composition of eye and } \\
\text { for cosmetics (named } \\
\text { Kohls in Egypt and } \\
\text { Sormea in Iran) }\end{array}$ & $\begin{array}{l}\text { Eaten fresh, } \\
\text { Powder eaten, } \\
\text { burned }\end{array}$ & 16 & 3.99 \\
\hline \multicolumn{8}{|c|}{ Rutaceae } \\
\hline 44 & $\begin{array}{l}\text { Citrus bergamia } \\
\text { Risso }\end{array}$ & $\begin{array}{l}\text { /li:mu: Ji:ri:n/ } \\
\text { ليموشيرين } \\
\text { Limoshirin (Az) } \\
\text { Bergamot (TR) }\end{array}$ & Fruit & $\begin{array}{l}\text { Improve blood } \\
\text { circulation, lipid and } \\
\text { cholesterol lowering }\end{array}$ & Eaten fresh & 19 & 4.73 \\
\hline \multicolumn{8}{|c|}{ Salicaceae } \\
\hline \multirow[t]{2}{*}{45} & Salix aegyptiaca L. & $\begin{array}{l}\text { /bi:dmefk/ } \\
\text { بيدمشَ } \\
\text { Bidmeshk (AZ) } \\
\text { Söğüt (TR) }\end{array}$ & Flower & $\begin{array}{l}\text { Heat exhaustion and } \\
\text { sedative }\end{array}$ & $\begin{array}{l}\text { Distillate, } \\
\text { decoction }\end{array}$ & 18 & 4.48 \\
\hline & & & & & & 401 & 100 \\
\hline
\end{tabular}

Note: Authorities to all scientific species names has been validated by www.theplantlist.org; Turkish names of herbs have been added according to http://www.dogaltedavi.net/bitkiisimlerilt.html.

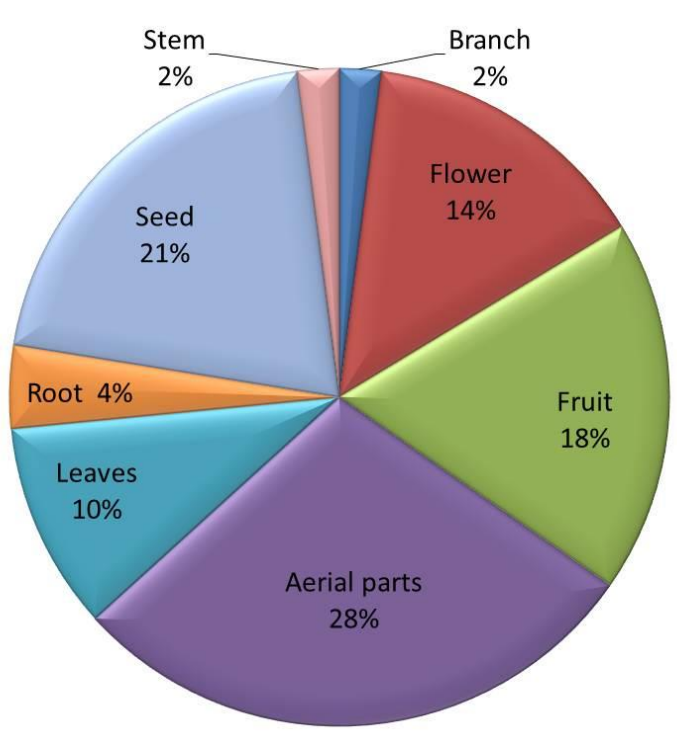

Figure 2. Plant parts used for the management of various healthcare problems in the villages of Qorveh-e Darjazin district, Hamedan Iran

Table 2. Plant parts used for preparation of the 49 herbal remedies from the 45 medicinal plant species growing in in the study area

\begin{tabular}{lcc}
\hline Plant parts used (A-Z) & $\begin{array}{c}\text { Absolute } \\
\text { value }\end{array}$ & $\begin{array}{c}\text { Frequency } \\
\text { (\%) }\end{array}$ \\
\hline Branch & 1 & 2.04 \\
Flower & 7 & 14.28 \\
Fruit & 9 & 18.36 \\
Gum & 0 & 0 \\
Aerial parts & 14 & 28.57 \\
Latex & 0 & 0 \\
Leaves & 5 & 10.20 \\
Root & 2 & 4.08 \\
Seed & 10 & 20.40 \\
Shoot & 0 & 0 \\
Stem & 1 & 2.04 \\
\hline Total & 49 & 100 \\
\hline
\end{tabular}

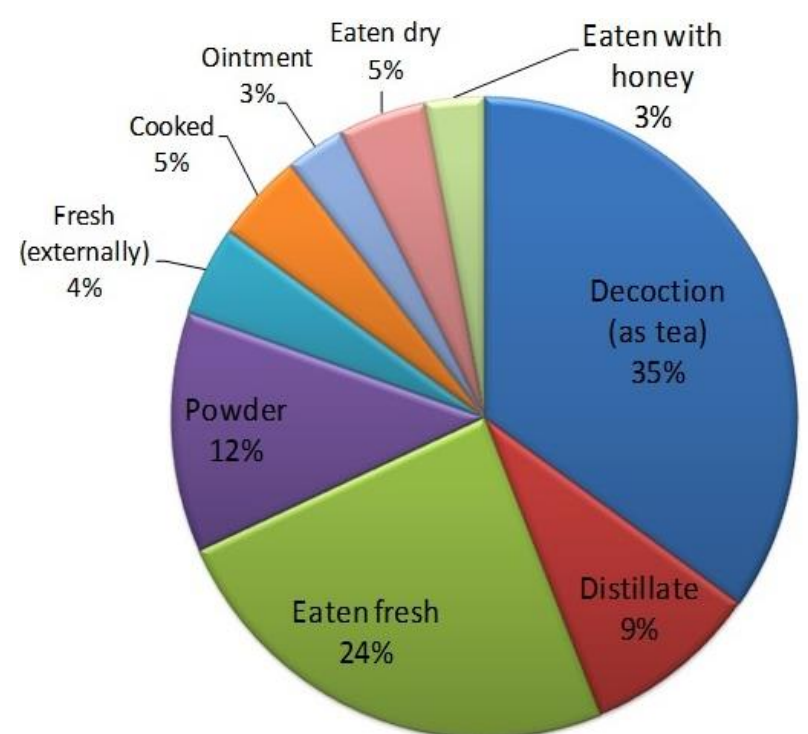

Figure 3. Local forms of crude drug preparation from medicinal plant/part(s) in the study area

Table 3. Local forms of preparation and application of the 49 herbal remedies from 45 medicinal plant species growing in the study area

\begin{tabular}{lcc}
\hline Medicinal preparation & $\begin{array}{c}\text { Absolute } \\
\text { value }\end{array}$ & $\begin{array}{c}\text { Frequency } \\
\text { (\%) }\end{array}$ \\
\hline Decoction (as tea) & 23 & 34.84 \\
Distillate & 6 & 9.09 \\
Decoction (as gargle) & 0 & 0 \\
Eaten fresh & 16 & 24.24 \\
Powder & 8 & 12.12 \\
Fresh (externally) & 3 & 4.54 \\
Cooked & 3 & 4.54 \\
Ointment & 2 & 3.03 \\
Eaten dry & 3 & 4.54 \\
Smoke & 0 & 0 \\
Suppository & 0 & 0 \\
Eaten with honey & 2 & 3.03 \\
\hline Total & 66 & 100 \\
\hline
\end{tabular}


Table 4. Most used medicinal plant species and their formulation as mono, di and Polyherbal for maximum usage in the treatment of various ailments in the study area

\begin{tabular}{|c|c|c|c|c|}
\hline \multicolumn{2}{|c|}{ Medicinal plants species } & \multirow{2}{*}{ Medicinal uses for ailments } & \multicolumn{2}{|c|}{$\begin{array}{l}\text { Acceptance rate of di and Polyherbal formulation of medicinal plants species by } 87 \text { informants of } 28 \text { villages ( } A=B e s t ; B=\text { Very } \\
\text { good; } C=\text { Good; } D=L e s s \text { used; E=Don't know) plus preparation methods of plants }\end{array}$} \\
\hline Absolute value & Voucher specimen & & Diherbal formulation & Polyherbal formulation \\
\hline Jaundice & Avena sativa L. & Jaundice treatment & -- & -- \\
\hline Wind & Carum carvi L. & Trapped gas or wind pain & -- & -- \\
\hline \multirow{2}{*}{$\begin{array}{l}\text { Bone and } \\
\text { rheumatic } \\
\text { disorders }\end{array}$} & Brassica oleracea L. & Bone diseases and gout & \multirow{2}{*}{$\begin{array}{l}\text { P. mungo + B. oleracea }(\text { Eaten cooked; } \mathrm{A}=23 \% ; \mathrm{B}=40.23 \% \text {; } \\
\mathrm{C}=17.2 \% ; \mathrm{D}=9.2 \% ; \mathrm{E}=10.3 \%)\end{array}$} & \multirow{2}{*}{--} \\
\hline & Phaseolus mungo L. & $\begin{array}{l}\text { Increasing blood and bone loss } \\
\text { treatment }\end{array}$ & & \\
\hline \multirow{2}{*}{ Hypertension } & Allium sativum L. & High blood pressure lowering & \multirow{2}{*}{$\begin{array}{l}\text { Morus nigra L. + Allium sativum L. (Eaten as fresh and } \\
\text { powder; Acceptance rate: } A=33.3 \% ; B=26.4 \% ; C=19.5 \% ; \\
D=11.5 \% ; E=3.4 \%)\end{array}$} & \multirow[t]{2}{*}{--} \\
\hline & Morus nigra L. & High blood pressure lowering & & \\
\hline \multirow{2}{*}{ Arrhythmia } & Mentha spp. & $\begin{array}{l}\text { Cardiovascular diseases, diarrhea, } \\
\text { heat exhaustion and sedative }\end{array}$ & \multirow{2}{*}{$\begin{array}{l}\text { B. officinalis + Mentha spp. (Decoction; Acceptance rate: } \\
\text { A=f6.1\%; } B=27.5 \% ; C=32.2 \% ; D=19.5 \% ; E=4.6 \%)\end{array}$} & \multirow{2}{*}{--} \\
\hline & Borago officinalis L. & $\begin{array}{l}\text { Blood purifier, dissolve kidney } \\
\text { stones and heart arrhythmia }\end{array}$ & & \\
\hline \multirow{2}{*}{$\begin{array}{l}\text { Calming the } \\
\text { nervous systems }\end{array}$} & Melissa officinalis L. & $\begin{array}{l}\text { Calming the nervous system and } \\
\text { schizophrenia }\end{array}$ & \multirow{2}{*}{$\begin{array}{l}\text { M. officinalis + S. aegyptiaca (Distillate; Acceptance rate: } \\
\mathrm{A}=43.6 \% ; \mathrm{B}=31 \% ; \mathrm{C}=32.2 \% ; \mathrm{D}=19.5 \% ; \mathrm{E}=4.6 \% \text { ) }\end{array}$} & \multirow{2}{*}{--} \\
\hline & Salix aegyptiaca L. & Heat exhaustion and sedative & & \\
\hline \multirow{2}{*}{$\begin{array}{l}\text { Irregular } \\
\text { menstruation }\end{array}$} & $\begin{array}{l}\text { Thymus kotschyanus Boiss. } \\
\text { \& Hohen. }\end{array}$ & $\begin{array}{l}\text { Diarrhea and bowel infections and } \\
\text { menstrual irregularities, heat } \\
\text { exhaustion and sedative }\end{array}$ & \multirow{2}{*}{$\begin{array}{l}\text { T. kotschyanus + A. eriophora (Eaten as powder, decoction } \\
\text { and distillate; Acceptance rate: } \mathrm{A}=40.2 \% ; \mathrm{B}=33.3 \% ; \mathrm{C}=17.2 \% \text {; } \\
\mathrm{D}=4.5 \% ; \mathrm{E}=4.5 \% \text { ) }\end{array}$} & \multirow[t]{2}{*}{--} \\
\hline & Achillea eriophora DC. & $\begin{array}{l}\text { Itching and menstrual } \\
\text { irregularities }\end{array}$ & & \\
\hline \multirow{2}{*}{ Diarrhea } & $\begin{array}{l}\text { Thymus kotschyanus Boiss. } \\
\text { \& Hohen. }\end{array}$ & $\begin{array}{l}\text { Diarrhea and bowel infections and } \\
\text { menstrual irregularities, heat } \\
\text { exhaustion and sedative }\end{array}$ & \multirow{2}{*}{$\begin{array}{l}\text { T. kotschyanus + Mentha spp. (Powder fresh, decoction, } \\
\text { Distillate, Leaves and Branch; Acceptance rate: } A=47.1 \% \text {; } \\
\mathrm{B}=31 \% ; \mathrm{C}=11.5 \% ; \mathrm{D}=4.5 \% ; \mathrm{E}=5.7 \% \text { ) }\end{array}$} & \multirow{2}{*}{--} \\
\hline & Mentha spp. & $\begin{array}{l}\text { Cardiovascular diseases and } \\
\text { diarrhea and heat exhaustion and } \\
\text { sedative }\end{array}$ & & \\
\hline \multirow{2}{*}{ Eye reinforcement } & Geranium spp. & $\begin{array}{l}\text { Eye movements and } \\
\text { reinforcement, lung disease, heat }\end{array}$ & \multirow{2}{*}{$\begin{array}{l}\text { Geranium spp. + O. dictamnus (Decoction or distillate; } \\
\text { Acceptance rate: } A=22.98 \% ; B=26.43 \% ; C=35.63 \% ; D=3.44 \% ; \\
E=11.49 \% \text { ) }\end{array}$} & \multirow[b]{2}{*}{--} \\
\hline & Origanum dictamnus L. & $\begin{array}{l}\text { exhaustion, } \\
\text { Sedative, eye movements and } \\
\text { reinforcement }\end{array}$ & & \\
\hline \multirow{3}{*}{$\begin{array}{l}\text { Stomach aches, } \\
\text { gastric ulcers, and } \\
\text { heartburn }\end{array}$} & Conium maculatum L. & $\begin{array}{l}\text { Stomach aches, gastric ulcers, and } \\
\text { heartburn }\end{array}$ & \multirow{3}{*}{$\begin{array}{l}\text { A. officinalis + C. maculatum (Decoction; Acceptance rate: } \\
A=34.48 \% ; B=27.58 \% ; C=22.98 \% ; D=8.04 \% ; E=6.89 \%) \\
\text { A. officinalis + Beta vulgaris L. (Decoction; Acceptance rate: } \\
A=27.58 \% ; B=36.78 \% ; C=22.98 \% ; D=5.74 \% ; E=6.89 \%)\end{array}$} & \multirow{3}{*}{-- } \\
\hline & Althea officinalis & Cold and fever & & \\
\hline & Beta vulgaris $\mathrm{L}$. & Cold, fever, chest pain & & \\
\hline \multirow{2}{*}{$\begin{array}{l}\text { Lipid and } \\
\text { cholesterol } \\
\text { lowering }\end{array}$} & Rhus coriaria L. & $\begin{array}{l}\text { Lipid and cholesterol lowering and } \\
\text { Blood purifier }\end{array}$ & \multirow{2}{*}{$\begin{array}{l}\text { C. bergamia Risso + C. intybus (Juice + decoction; Acceptance } \\
\text { rate: } A=29.88 \% ; B=29.88 \% ; C=17.24 \% ; D=13.79 \% ; E=9.19 \% \text { ) } \\
\text { Citrus bergamia Risso + C. intybus (Juice + decoction; }\end{array}$} & \multirow{2}{*}{--} \\
\hline & Citrus bergamia Risso & $\begin{array}{l}\text { Lipid and cholesterol lowering and } \\
\text { Blood purifier }\end{array}$ & & \\
\hline
\end{tabular}

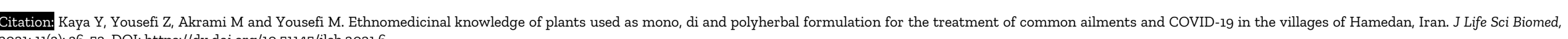
2021; 11(3): 36-52. DOI: https://dx.doi.org/10.51145/jlsb.2021.6 


\begin{tabular}{|c|c|c|c|c|}
\hline & Cichorium intybus L. & $\begin{array}{l}\text { Blood purifier and removal } \\
\text { Heatstroke and sedative, lipid and } \\
\text { cholesterol lowering }\end{array}$ & $\begin{array}{l}\text { Acceptance rate: } A=20.68 \% ; B=33.33 \% ; C=14.94 \% ; \\
D=22.98 \% ; E=8.04 \%)\end{array}$ & \\
\hline \multirow{3}{*}{ Anemia } & Spinacia oleracea L. & Treatment of anemia & \multirow{3}{*}{$\begin{array}{l}\text { S. oleracea L. + C. arietinum (Eaten as cooked; Acceptance } \\
\text { rate: } A=27.58 \% ; B=21.83 \% ; C=24.13 \% ; D=17.24 \% ; E=9.19 \%) \\
\text { S. oleracea + P. mungo (Eaten as cooked; Acceptance rate: } \\
A=22.98 \% ; B=28.73 \% ; C=17.24 \% ; D=20.68 \% ; E=10.34 \% \text { ) } \\
\text { C. arietinum + P. mungo (Eaten as cooked; Acceptance rate: } \\
A=19.54 \% ; B=17.24 \% ; C=32.18 \% ; D=12.64 \% ; E=18.39 \%) \\
\text { S. oleracea L. + P. mungo (Eaten as cooked; Acceptance rate: } \\
A=20.68 \% ; B=33.33 \% ; C=14.94 \% ; D=22.98 \% ; E=8.04 \%)\end{array}$} & \multirow[t]{3}{*}{$\begin{array}{l}\text { S. oleracea + C. arietinum + P. mungo (Eaten as cooked; Acceptance rate: } \mathrm{A}=34.48 \% ; \\
\mathrm{B}=31.03 \% ; \mathrm{C}=18.39 \% ; \mathrm{D}=9.19 \% ; \mathrm{E}=6.89 \%), 78 \%\end{array}$} \\
\hline & Cicer arietinum L. & Increasing blood iron levels & & \\
\hline & Phaseolus mungo L. & $\begin{array}{l}\text { Increasing blood and bone loss } \\
\text { treatment }\end{array}$ & & \\
\hline \multirow{4}{*}{ Blood purifier } & Berberis integerrima L. & $\begin{array}{l}\text { Blood cleanser to cure pimples and } \\
\text { acne }\end{array}$ & \multirow{4}{*}{$\begin{array}{l}\text { B. integerrima + P. granatum (Juice; Acceptance rate: } \\
A=47.12 \% ; B=32.18 \% ; C=11.49 \% ; D=5.74 \% ; E=3.44 \% \text { ) } \\
\text { B. integerrima + B. officinalis (Decoction; Acceptance rate: } \\
A=43.67 \% ; B=28.73 \% ; C=13.79 \% ; D=5.74 \% ; E=8.04 \% \text { ) } \\
\text { C. bergamia Risso + B. officinalis (Juice + decoction; } \\
\text { Acceptance rate: } A=25.28 \% ; B=20.68 \% ; C=26.43 \% ; \\
D=16.09 \% ; E=11.49 \% \text { ) }\end{array}$} & \multirow[t]{4}{*}{$\begin{array}{l}\text { B. integerrima + P. granatum + B. officinalis = (Juice + decoction; Acceptance rate: } \\
\mathrm{A}=35.63 \% ; \mathrm{B}=33.33 \% ; \mathrm{C}=10.34 \% ; \mathrm{D}=12.64 \% ; \mathrm{E}=8.04 \%)\end{array}$} \\
\hline & Citrus bergamia Risso & $\begin{array}{l}\text { Smooth blood, lipid and cholesterol } \\
\text { lowering }\end{array}$ & & \\
\hline & Punica granatum L. & Blood purifier & & \\
\hline & Borago officinalis L. & $\begin{array}{l}\text { Blood purifier and dissolve kidney } \\
\text { stones + heart arrhythmia }\end{array}$ & & \\
\hline \multirow{4}{*}{$\begin{array}{l}\text { Increasing } \\
\text { intelligence } \\
\text { quotient (IQ), the } \\
\text { sexual power, } \\
\text { sperm count and } \\
\text { motility, and } \\
\text { cosmetics uses }\end{array}$} & Muscari comosum (L.) Mill. & $\begin{array}{l}\text { Improving sexual stamina; } \\
\text { boosting semen volume, sperm } \\
\text { count and motility; }\end{array}$ & \multirow{4}{*}{$\begin{array}{l}\text { J. regia + A. communis L. (Seed eaten fresh or dry; } \\
\text { Acceptance rate: } A=34.48 \% ; B=36.78 \% ; C=22.98 \% ; D=5.74 \% ; \\
E=0 \%) \\
\text { J. regia + C. avellana L. (Seed eaten fresh or dry; Acceptance } \\
\text { rate: Acceptance rate: } A=35.63 \% ; B=35.63 \% ; C=21.83 \% ; \\
D=6.89 \% ; E=0 \% \text { ) } \\
\text { C. avellana L. + A. communis L. (Seed eaten fresh or dry; } \\
\text { Acceptance rate: } A=33.33 \% ; B=33.33 \% ; C=32.18 \% ; D=1.14 \% ; \\
E=0 \%)\end{array}$} & \multirow{4}{*}{$\begin{array}{l}\text { J. regia + A. communis + C. avellana (Eaten as nuts and powder; Acceptance rate: } \\
A=45.97 \% ; B=42.52 \% ; C=11.49 \% ; D=0 \% ; E=0 \%) \\
\text { M. comosum + J. regia + A. communis + C. avellana (Eaten as powder mixed with honey; } \\
\text { Acceptance rate: } A=42.52 \% ; B=44.82 \% ; C=5.74 \% ; D=5.74 \% ; E=1.14 \%)\end{array}$} \\
\hline & Juglans regia L. & \multirow{3}{*}{$\begin{array}{l}\text { Improving sexual stamina; } \\
\text { boosting semen volume, sperm } \\
\text { count and motility; enhancing } \\
\text { intelligence; composition of eye } \\
\text { cosmetics (named Kohls in Cairo } \\
\text { city, Egypt or Sormea in Iran) }\end{array}$} & & \\
\hline & Amygdalus communis L. & & & \\
\hline & Corylus avellana L. & & & \\
\hline \multirow{4}{*}{ Cold and fever } & Althea officinalis & Cold, fever and chest pain & \multirow{4}{*}{$\begin{array}{l}\text { A. officinalis + B. vulgaris L. (Decoction; Acceptance rate: } \\
\mathrm{A}=27.58 \% ; \mathrm{B}=25.28 \% ; \mathrm{C}=20.68 \% ; \mathrm{D}=17.24 \% ; \mathrm{E}=10.34 \%) \\
\text { B. vulgaris + B. rapa (Decoction of root; Acceptance rate: } \\
\mathrm{A}=25.28 \% ; \mathrm{B}=22.98 \% ; \mathrm{C}=22.98 \% ; \mathrm{D}=16.09 \% ; \mathrm{E}=12.64 \%) \\
\text { A. officinalis + M. sylvestris (Decoction; Acceptance rate: } \\
\mathrm{A}=34.48 \% ; \mathrm{B}=28.73 \% ; \mathrm{C}=17.24 \% ; \mathrm{D}=11.49 \% ; \mathrm{E}=8.04 \%) \\
\text { M. sylvestris + B. rapa (Decoction; Acceptance rate: } \\
\mathrm{A}=22.98 \% ; \mathrm{B}=20.68 \% ; \mathrm{C}=36.78 \% ; \mathrm{D}=10.34 \% ; \mathrm{E}=9.19 \%)\end{array}$} & \multirow{4}{*}{$\begin{array}{l}\text { A. officinalis + M. sylvestris + B. rapa = (Decoction; Acceptance rate: } A=27.58 \% ; B=33.33 \% ; \\
C=22.98 \% ; D=6.89 \% ; E=9.19 \%) \\
\text { A. officinalis }+ \text { M. sylvestris + B. vulgaris = (Decoction; Acceptance rate: } A=32.18 \% ; \\
B=34.48 \% ; C=20.68 \% ; D=5.74 \% ; E=6.89 \%) \\
\text { A. officinalis }+ \text { M. sylvestris + B. rapa + B. vulgaris = (Decoction; Acceptance rate: } A=32.18 \% ; \\
\text { B=36.78\%; } C=13.79 \% ; D=9.19 \% ; E=8.04 \%)\end{array}$} \\
\hline & Beta vulgaris L. & Cold, fever and chest pain & & \\
\hline & Malva sylvestris L. & Laxative and fever treatment & & \\
\hline & Brassica rapa L. & $\begin{array}{l}\text { Laxative, fever, catarrh and chest } \\
\text { pain }\end{array}$ & & \\
\hline \multirow{4}{*}{$\begin{array}{l}\text { Flu and lung } \\
\text { diseases }\end{array}$} & Origanum dictamnus $\mathrm{L}$. & $\begin{array}{l}\text { Lung disease, heat exhaustion and } \\
\text { sedative, eye movements and } \\
\text { reinforcement }\end{array}$ & \multirow{4}{*}{$\begin{array}{l}\text { B. rapa + B. vulgaris (Decoction; Acceptance rate: } \mathrm{A}=25.28 \% \text {; } \\
\mathrm{B}=22.98 \% ; \mathrm{C}=31.03 \% ; \mathrm{D}=11.49 \% ; \mathrm{E}=9.19 \%) \\
\text { A. officinalis + B. vulgaris (Decoction; Acceptance rate: } \\
\mathrm{A}=22.98 \% ; \mathrm{B}=20.68 \% ; \mathrm{C}=35.63 \% ; \mathrm{D}=11.49 \% ; \mathrm{E}=9.19 \%)\end{array}$} & \multirow{4}{*}{$\begin{array}{l}\text { A. officinalis + B. vulgaris + B. rapa (Decoction; Acceptance rate: } A=41.37 \% ; B=42.52 \% ; \\
C=11.49 \% ; D=4.59 \% ; E=0 \%) \\
\text { A. officinalis + B. vulgaris + B. rapa + O. dictamnus L. (Decoction, decoction + distillate; } \\
\text { Acceptance rate: } A=42.52 \% ; B=41.37 \% ; C=11.49 \% ; D=4.59 \% ; E=0 \%)\end{array}$} \\
\hline & Althea officinalis & Cold and fever & & \\
\hline & Brassica rapa L. & $\begin{array}{l}\text { Laxative, fever, catarrh and chest } \\
\text { pain }\end{array}$ & & \\
\hline & Beta vulgaris L. & Cold, chest pain, fever & & \\
\hline
\end{tabular}




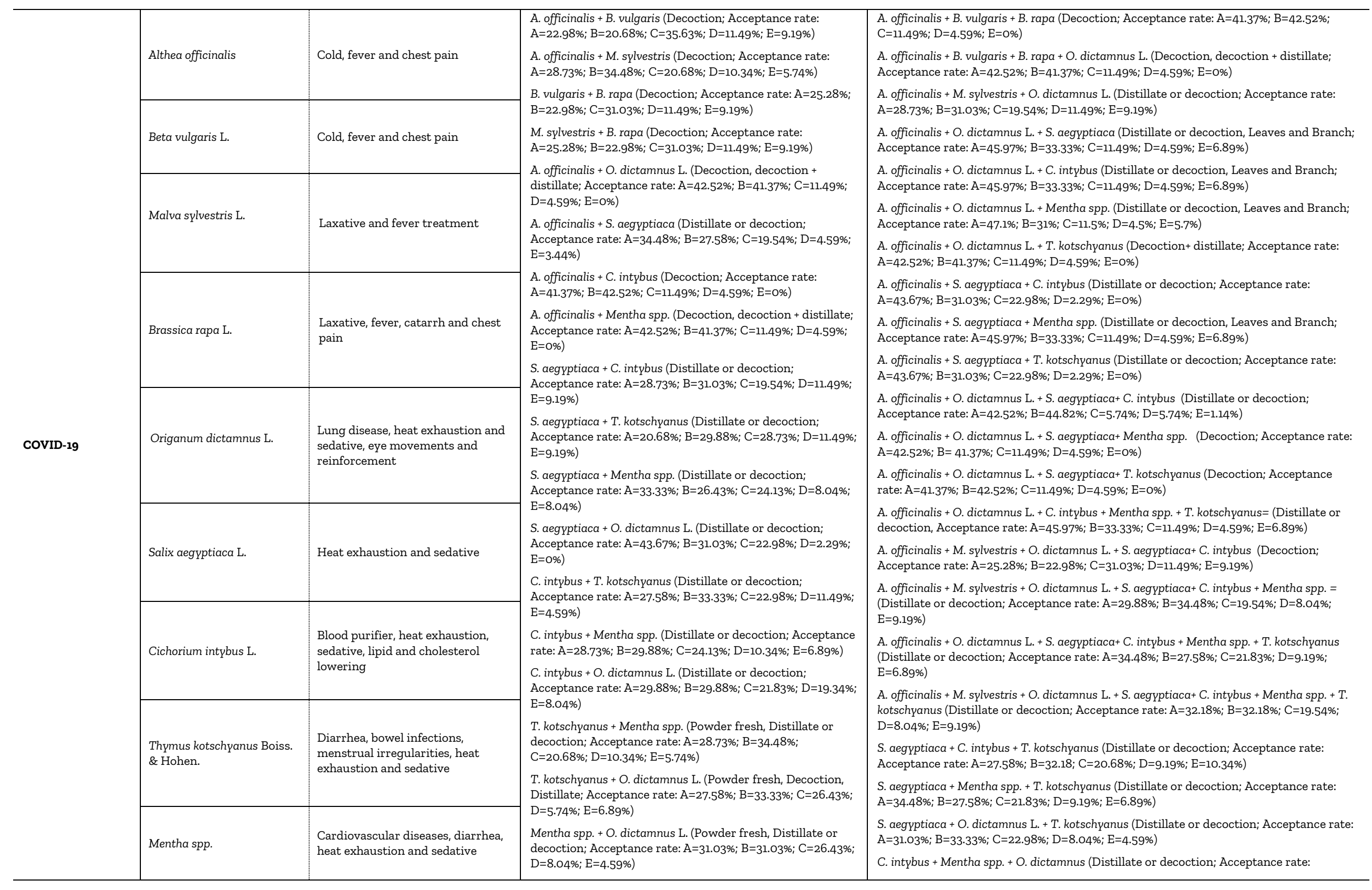




\begin{tabular}{|c|c|c|c|c|}
\hline & & & & $\mathrm{A}=27.58 \% ; \mathrm{B}=33.33 \% ; \mathrm{C}=22.98 \% ; \mathrm{D}=11.49 \% ; \mathrm{E}=4.59 \%)$ \\
\hline & & & & $\begin{array}{l}\text { C. intybus + Mentha spp. + T. kotschyanus (Distillate or decoction; Acceptance rate: } \\
\mathrm{A}=34.48 \% ; \mathrm{B}=27.58 \% ; \mathrm{C}=19.54 \% ; \mathrm{D}=4.59 \% ; \mathrm{E}=3.44 \% \text { ) }\end{array}$ \\
\hline & & & & $\begin{array}{l}\text { T. kotschyanus + Mentha spp. + O. dictamnus (Powder fresh, Decoction, Distillate, Leaves } \\
\text { and Branch; Acceptance rate: } A=22.98 \% ; B=34.48 \% ; C=23 \% ; D=8 \% ; E=11.49 \% \text { ) }\end{array}$ \\
\hline & & & & $\begin{array}{l}\text { S. aegyptiaca + C. intybus + T. kotschyanus + Mentha spp. (Distillate or decoction, Leaves } \\
\text { and Branch; Acceptance rate: } A=31.03 \% ; B=28.73 \% ; C=20.68 \% ; D=10.34 \% ; E=10.34 \%)\end{array}$ \\
\hline & & & & $\begin{array}{l}\text { S. aegyptiaca }+C \text {. intybus + T. kotschyanus + O. dictamnus (Distillate or decoction, Leaves } \\
\text { and Branch; Acceptance rate: } A=28.73 \% ; B=29.88 \% ; C=20.68 \% ; D=11.49 \% ; E=9.19 \% \text { ) }\end{array}$ \\
\hline & & & & $\begin{array}{l}\text { S. aegpptiaca + T. kotschyanus + Mentha spp. + O. dictamnus (Distillate or decoction, } \\
\text { Leaves and Branch; Acceptance rate: } A=32.18 \% ; B=32.18 \% ; C=19.54 \% ; D=8.04 \% ; E=9.19 \% \text { ) }\end{array}$ \\
\hline & & & & $\begin{array}{l}\text { S. aegyptiaca + C. intybus + Mentha spp. + O. dictamnus (Distillate or decoction, Leaves } \\
\text { and Branch; Acceptance rate: } \mathrm{A}=29.88 \% ; \mathrm{B}=34.48 \% ; \mathrm{C}=19.54 \% ; \mathrm{D}=8.04 \% ; \mathrm{E}=9.19 \% \text { ) }\end{array}$ \\
\hline & & & & $\begin{array}{l}\text { C. intybus }+ \text { T. kotschyanus }+ \text { Mentha spp. }+ \text { O. dictamnus (Distillat or Decoction, Leaves } \\
\text { and Branch; Acceptance rate: } A=27.58 \% ; B=35.63 \% ; C=22.98 \% ; D=8.04 \% ; E=5.74 \% \text { ) }\end{array}$ \\
\hline & & & & $\begin{array}{l}\text { S. aegyptiaca + C. intybus + T. kotschyanus + Mentha spp. + O. dictamnus (Distillat or } \\
\text { Decoction, Leaves and Branch; Acceptance rate: } \mathrm{A}=45.97 \% ; \mathrm{B}=33.33 \% ; \mathrm{C}=11.49 \% ; \\
\mathrm{D}=4.59 \% ; \mathrm{E}=6.89 \% \text { ) }\end{array}$ \\
\hline \multirow{11}{*}{ Sunstroke } & & & \multirow{11}{*}{ 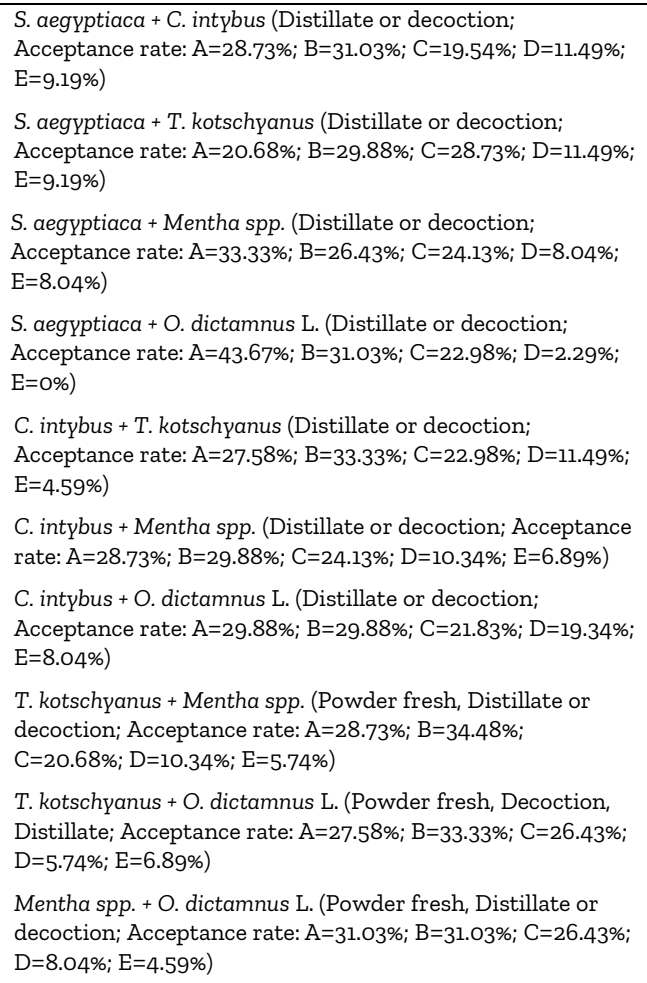 } & $\begin{array}{l}\text { S. aegyptiaca + C. intybus + T. kotschyanus (Distillate or decoction; Acceptance rate: } \\
\text { Acceptance rate: } A=27.58 \% ; B=32.18 ; C=20.68 \% ; D=9.19 \% ; E=10.34 \% \text { ) }\end{array}$ \\
\hline & Salix aegyptiaca L. & Heat exhaustion and sedative & & $\begin{array}{l}\text { S. aegyptiaca + Mentha spp. + T. kotschyanus (Distillate or decoction; Acceptance rate: } \\
\mathrm{A}=34.48 \% ; \mathrm{B}=27.58 \% ; \mathrm{C}=21.83 \% ; \mathrm{D}=9.19 \% ; \mathrm{E}=6.89 \% \text { ) }\end{array}$ \\
\hline & & & & $\begin{array}{l}\text { S. aegyptiaca + O. dictamnus L. + T. kotschyanus (Distillate or decoction; Acceptance rate: } \\
A=31.03 \% ; B=33.33 \% ; C=22.98 \% ; D=8.04 \% ; E=4.59 \% \text { ) }\end{array}$ \\
\hline & Cichorium intybus L. & $\begin{array}{l}\text { Blood purifier, heat exhaustion, } \\
\text { sedative, lipid and cholesterol }\end{array}$ & & $\begin{array}{l}\text { C. intybus + Mentha spp. + O. dictamnus (Distillate or decoction; Acceptance rate: } \\
\mathrm{A}=27.58 \% ; \mathrm{B}=33.33 \% ; \mathrm{C}=22.98 \% ; \mathrm{D}=11.49 \% ; \mathrm{E}=4.59 \% \text { ) }\end{array}$ \\
\hline & & lowering & & $\begin{array}{l}\text { C. intybus + Mentha spp. + T. kotschyanus (Distillate or decoction; Acceptance rate: } \\
\mathrm{A}=34.48 \% ; \mathrm{B}=27.58 \% ; \mathrm{C}=19.54 \% ; \mathrm{D}=4.59 \% ; \mathrm{E}=3.44 \% \text { ) }\end{array}$ \\
\hline & $\begin{array}{l}\text { Thymus kotschyanus Boiss. } \\
\text { \& Hohen. }\end{array}$ & $\begin{array}{l}\text { Diarrhea, bowel infections, } \\
\text { menstrual irregularities, heat }\end{array}$ & & $\begin{array}{l}\text { T. kotschyanus + Mentha spp. + O. dictamnus (Powder fresh, Decoction, Distillate, Leaves } \\
\text { and Branch; Acceptance rate: } A=22.98 \% ; B=34.48 \% ; C=23 \% ; D=8 \% ; E=11.49 \% \text { ) } \\
\text { S. aegyptiaca + C. intybus + T. kotschyanus + Mentha spp. (Distillate or decoction, Leaves } \\
\text { and Branch; Acceptance rate: } A=31.03 \% ; B=28.73 \% ; C=20.68 \% ; D=10.34 \% ; E=10.34 \%)\end{array}$ \\
\hline & & & & $\begin{array}{l}\text { S. aegyptiaca }+ \text { C. intybus + T. kotschyanus + O. dictamnus (Distillate or decoction, Leaves } \\
\text { and Branch; Acceptance rate: } A=28.73 \% ; B=29.88 \% ; C=20.68 \% ; D=11.49 \% ; E=9.19 \% \text { ) }\end{array}$ \\
\hline & & & & $\begin{array}{l}\text { S. aegyptiaca }+ \text { T. kotschyanus }+ \text { Mentha spp. }+ \text { O. dictamnus (Distillate or decoction, } \\
\text { Leaves and Branch; Acceptance rate: } A=32.18 \% ; B=32.18 \% ; C=19.54 \% ; D=8.04 \% ; E=9.19 \% \text { ) }\end{array}$ \\
\hline & Mentha spp. & heat exhaustion and sedative & & $\begin{array}{l}\text { S. aegyptiaca + C. intybus + Mentha spp. + O. dictamnus (Distillate or decoction, Leaves } \\
\text { and Branch; Acceptance rate: } \mathrm{A}=29.88 \% ; \mathrm{B}=34.48 \% ; \mathrm{C}=19.54 \% ; \mathrm{D}=8.04 \% ; \mathrm{E}=9.19 \%)\end{array}$ \\
\hline & & & & $\begin{array}{l}\text { C. intybus + T. kotschyanus + Mentha spp. + O. dictamnus (Distillate or decoction, Leaves } \\
\text { and Branch; Acceptance rate: } \mathrm{A}=27.58 \% ; \mathrm{B}=35.63 \% ; \mathrm{C}=22.98 \% ; \mathrm{D}=8.04 \% ; \mathrm{E}=5.74 \% \text { ) }\end{array}$ \\
\hline & Origanum dictamnus $\mathrm{L}$. & $\begin{array}{l}\text { Lung disease, heat exhaustion, } \\
\text { Sedative, eye movements and } \\
\text { reinforcement }\end{array}$ & & $\begin{array}{l}\text { S. aegyptiaca + C. intybus + T. kotschyanus + Mentha spp. + O. dictamnus (Distillat or } \\
\text { Decoction, Leaves and Branch; Acceptance rate: } \mathrm{A}=45.97 \% ; \mathrm{B}=33.33 \% ; \mathrm{C}=11.49 \% ; \\
\mathrm{D}=4.59 \% ; \mathrm{E}=6.89 \% \text { ) }\end{array}$ \\
\hline
\end{tabular}

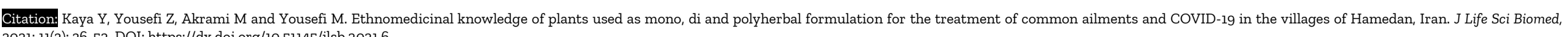
2021; 11(3): 36-52. DOI: https://dx.doi.org/10.51145/jlsb.2021.6 


\begin{tabular}{|c|c|c|c|c|}
\hline \multirow{6}{*}{ Skin disorders } & Achillea eriophora DC. & $\begin{array}{l}\text { Itching and menstrual } \\
\text { irregularities }\end{array}$ & \multirow{6}{*}{ 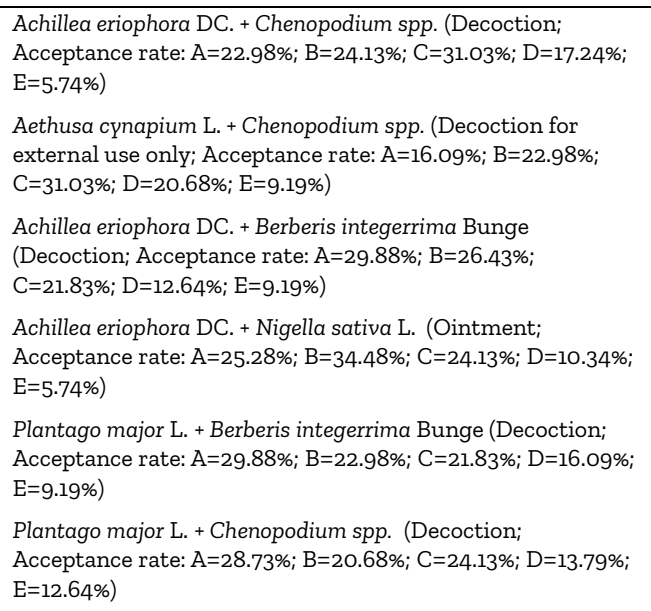 } & \multirow{6}{*}{$\begin{array}{l}\text { Plantago major L. + Berberis integerrima Bunge + Chenopodium spp. (Decoction; } \\
\text { Acceptance rate: } \mathrm{A}=29.88 \% ; \mathrm{B}=26.43 \% ; \mathrm{C}=21.83 \% ; \mathrm{D}=13.79 \% .64 \% ; \mathrm{E}=9.19 \%) \\
\text { Achillea eriophora } \mathrm{DC} \text {. + Chenopodium spp. + Berberis integerrima Bunge (Decoction; } \\
\text { Acceptance rate: } \mathrm{A}=20.68 \% ; \mathrm{B}=28.73 \% ; \mathrm{C}=20.68 \% ; \mathrm{D}=18.39 \% ; \mathrm{E}=11.49 \%) \\
\text { Achillea eriophora } \mathrm{DC} \text {. + Chenopodium spp. + Berberis integerrima Bunge + Aethusa } \\
\text { cynapium L. (Decoction for external use only; Acceptance rate: } \mathrm{A}=12.64 \% ; \mathrm{B}=21.83 \% ; \\
\mathrm{C}=28.73 \% ; \mathrm{D}=24.13 \% ; \mathrm{E}=12.64 \%)\end{array}$} \\
\hline & Aethusa cynapium L. & Animal bites and snake bites & & \\
\hline & Nigella sativa L. & Removing warts & & \\
\hline & Chenopodium spp. & Skin rash with itching & & \\
\hline & Berberis integerrima Bunge & $\begin{array}{l}\text { Blood cleanser to cure pimples and } \\
\text { acne }\end{array}$ & & \\
\hline & Plantago major L. & $\begin{array}{l}\text { Acne, skin softening and dispose of } \\
\text { excess fluids, gastrointestinal } \\
\text { bleeding }\end{array}$ & & \\
\hline \multirow{6}{*}{$\begin{array}{l}\text { Urinary system } \\
\text { disorders }\end{array}$} & Borago officinalis L. & $\begin{array}{l}\text { Blood purifier, dissolve kidney } \\
\text { stones s and heart arrhythmia }\end{array}$ & \multirow{6}{*}{$\begin{array}{l}\text { B. officinalis + Z. mays (Decoction; Acceptance rate: } \\
\text { A=11.49\%; } B=16.09 \% ; C=31.03 \% ; D=28.73 \% ; E=12.64 \%) \\
\text { B. officinalis + A. graveolens (Decoction; Acceptance rate: } \\
\text { A=f1.49\%; } B=18.39 \% ; C=31.03 \% ; D=26.43 \% ; E=12.64 \%) \\
\text { C. vulgaris Schrad. + Z. mays (Decoction; Acceptance rate: } \\
A=22.98 \% ; B=22.98 \% ; C=27.58 \% ; D=12.64 \% ; E=13.79 \%) \\
\text { T. kotschyanus + T. collinus (Eaten as fresh and cooked, } \\
\text { Decoction; Acceptance rate: } A=29.88 \% ; B=22.98 \% ; \\
C=22.98 \% ; D=13.79 \% ; E=10.34 \%)\end{array}$} & \multirow{6}{*}{$\begin{array}{l}\text { B. officinalis + Z. mays L. + A. graveolens (Decoction; Acceptance rate: } A=11.49 \% ; B=14.94 \% ; \\
C=32.18 \% ; D=29.88 \% ; E=11.49 \% \text { ) } \\
\text { B. officinalis + C. vulgaris Schrad. + Z. mays L. + A. graveolens (Decoction; Acceptance rate: } \\
A=13.79 \% ; B=17.24 \% ; C=22.98 \% ; D=27.58 \% ; E=18.39 \% \text { ) }\end{array}$} \\
\hline & Citrullus vulgaris Schrad. & Dissolve kidney stones & & \\
\hline & Zea mays L. & Strong diuretic & & \\
\hline & Apium graveolens L. & $\begin{array}{l}\text { Dissolve kidney stones or renal } \\
\text { calculi }\end{array}$ & & \\
\hline & $\begin{array}{l}\text { Thymus kotschyanus Boiss. } \\
\text { \& Hohen. }\end{array}$ & $\begin{array}{l}\text { Diarrhea, bowel infections, } \\
\text { menstrual irregularities, heat } \\
\text { exhaustion and sedative }\end{array}$ & & \\
\hline & Tragopogon collinus DC. & $\begin{array}{l}\text { Bowel infections, stomach aches, } \\
\text { gastric ulcers, and heartburn }\end{array}$ & & \\
\hline \multirow{9}{*}{$\begin{array}{l}\text { Digestive system } \\
\text { diseases }\end{array}$} & Anemone coronaria L. & Mouth ulcers & \multirow{9}{*}{ 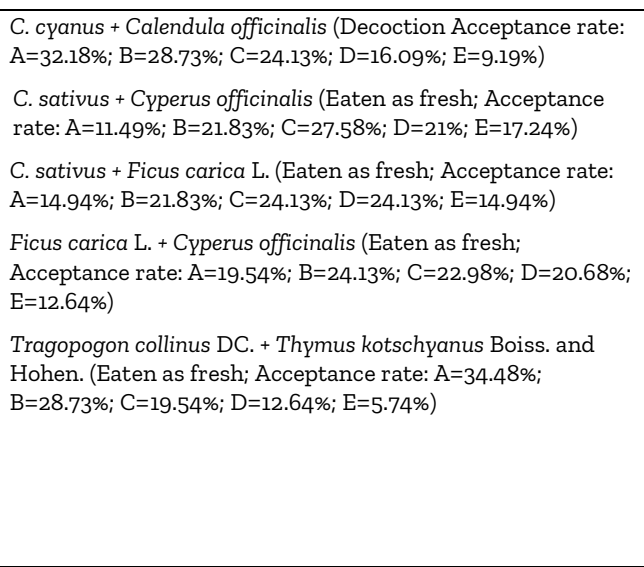 } & \multirow[t]{9}{*}{$\begin{array}{l}\text { C. sativus + CYperus officinalis + F. carica (Eaten as fresh, Acceptance rate: } A=17.24 \% ; \\
B=24.13 \% ; C=27.58 \% ; D=17.24 \% ; E=13.79 \% \text {; }\end{array}$} \\
\hline & $\begin{array}{l}\text { Astragalus adscendens } \\
\text { Boiss \& Hausskn. }\end{array}$ & $\begin{array}{l}\text { Swelling of the stomach and } \\
\text { intestines, hemorrhoids }\end{array}$ & & \\
\hline & Centaurium cyanus & \multirow{2}{*}{ Biliary excretion } & & \\
\hline & $\begin{array}{l}\text { Calendula officinalis } \mathrm{L} \text {. } \\
\text { Cucumis sativus } \mathrm{L} \text {. }\end{array}$ & & & \\
\hline & Cyperus officinalis $\mathrm{L}$. & $\begin{array}{l}\text { Constipation disposal, } \\
\text { hemorrhoids }\end{array}$ & & \\
\hline & Ficus carica $\mathrm{L}$. & $\begin{array}{l}\text { Chronic constipation disposal, } \\
\text { hemorrhoids }\end{array}$ & & \\
\hline & Tragopogon collinus DC. & $\begin{array}{l}\text { Bowel infections, stomach aches, } \\
\text { gastric ulcers, and heartburn }\end{array}$ & & \\
\hline & $\begin{array}{l}\text { Thymus kotschyanus Boiss. } \\
\text { \& Hohen. }\end{array}$ & $\begin{array}{l}\text { Diarrhea, bowel infections, } \\
\text { menstrual irregularities, heat } \\
\text { exhaustion and sedative }\end{array}$ & & \\
\hline & Plantago major L. & $\begin{array}{l}\text { Acne, skin softening and dispose of } \\
\text { excess fluids, gastrointestinal } \\
\text { bleeding, hemorrhoids }\end{array}$ & & \\
\hline
\end{tabular}

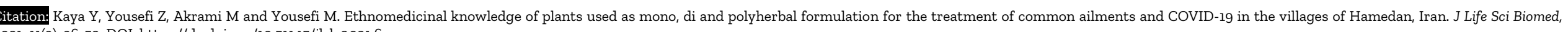
2021; 11(3): 36-52. DOI: https://dx.doi.org/10.51145/jlsb.2021.6 
Data obtained from other regions of Iran like Hormozgan [36], Kohgiluyeh and Boyer-Ahmad [28], Esfahan (Mobarakeh) [37], and Hamedan city [1, 34, 35, 38] showed that, Asteraceae, Lamiaceae and Apiaceae because of their wide range of biologically active compounds [39] were the most used families of medicinal plants for people's everyday health care needs.

The use's frequency of the different plant parts in herbal remedy preparation is shown in Table 2 and Figure 3. The results showed that the most widely parts of plant species used for medical purposes were the aerial parts, seed, fruit, flower and leaves, respectively. The higher frequency of use of herbs and leaves in herbal remedy preparation is largely due to an easily and readily availability of them [14]. The herbals are generally discovered by the method of isolation and purification, or knowledge on characterization of active ingredients and type of preparation. The term "herbal drug" determines the part(s) of a plant such as leaves, flowers, barks, stems, seeds, roots etc. used for preparing medicines [40]. Each part of the plants are utilized for the different pharmacological action and they may made into a range of herbal preparations including Plantago major $\mathrm{L}$. (Decoction), Melissa officinalis L. (Liquid Extract or distillate), Rhus coriaria L. (Powders), Cicer arietinum L. (cooked), Nigella sativa L. (Ointment) and etc. Özgen et al. [6] stated that distillate and decoctions are simple with more usable preparations and sufficient to make the active ingredients readily available without any further extraction [41].

The majority of human calories come from many edible seeds, especially from legumes, cereals and nuts [42]. Also, most cooking oils, beverages, spices and some important food additives are produced from seeds. While some seeds and or plants are edible, some of them are harmful or poisonous because of special chemical compounds to discourage plant herbivores and seed predators [26]. Many of these compounds are toxic or break down into toxic compounds within the digestive system and it can be more susceptible for children.

People who use folk medicinal remedies may not understand their scientific rationale, and they just rely on personal experience based on using therapeutic doses of some highly usable medicinal plants. Since there is a better understanding today of how the body functions, people are thus in a better position to understand the healing powers of medicinal plants and their potential as multi-functional chemical entities for treating complicated health conditions. Based on the available evidence, it can be concluded that the polyherbal combination of medicinal plants species are capable of possessing great medicinal usage of the wild and nonwild herbs used by people to cure various ailments.

Study performed by De Silva [43] on industrial utilization of medicinal plants in developing countries showed that the value of trade in herbal medicines has a yearly growth of $12-15 \%$. In fact, the world's population (about $80 \%$ ) still relies on their traditional medicines to cure the important and common ailments like digestive system diseases [44-48], liver disease [49, 50], urinary system disorders [51], skin disorders and rash [45, 52], diabetes [53], psychiatric disorders [54], lung diseases [41], pain relief [55], malaria [55] etc. [3, 28, 30, 57] for their everyday health care needs.

\section{COVID-19}

Results of table 4 are also revealed a high demand of people of this area to medicinal remedies as di and polyherbal formulations against the global pandemic coronavirus disease 2019 (COVID-19) that is a novel severe acute respiratory syndrome coronavirus 2 (SARS-CoV-2).

There is a crucial necessity for the effective drugs to tackle the virus outbreak. Since December 2020 up to now several drugs with existing pharmacological evidence are reported that may inhibit infection in COVID-19 patients [58-64]. Also, some types of coronavirus vaccines like Pfizer, Moderna, AstraZeneca, etc. are approved under an emergency use authorization by FDA approval [65]. But, since there are unknown and unique risks to messenger RNA vaccines, choosing the harmless substances like medicinal plants against COVID-19 is the most important issue in the treatment of suchlike infectious diseases, especially when its viral genome is currently mutated and cause a new variant or strain of a coronavirus like delta variant that is the most contagious and infectious version of the coronavirus worldwide.

Although currently there are no any research supports the use of any supplement to protect against COVID-19 specifically, but the repurposing of natural compounds may provide alternatives against COVID-19. Many people use medicinal plants, fruits, and nutraceuticals rich in $\mathrm{Zn}$, selenium, vitamin $\mathrm{D}$, vitamin $\mathrm{C}$, curcumin, cinnamaldehyde, quercetin, probiotics, lactoferrin, etc. have a proven ability of immune-boosting, antioxidant, anti-inflammatory and antiviral effects [66, 67]. 
Hence, there is a need of people of the global majority to phytonutrients and medicinal plants contain phytochemicals in a form of polyherbal formulation to boost the immune system, prevent the disease progress to a severe stage, and further suppress the hyper inflammation and therapeutic support against COVID-19.

\section{CONCLUSION}

The results of this study showed the high herbal diversity of medicinal plants in the studied area. However, the subsequent works should be aimed to help the phytomedicines studies, standardization of medicinal plant usage and safety in primary health care. From the ethnobotanical survey carried out in Qorveh-e Darjazin district, it can be concluded that local traditions surrounding the use of plants in folk medicine are still extensive and quite varied. These findings suggest that the polyherbal medicine is a useful tool for providing various interpretations to ethnobotanical knowledge in the intra- and intercommunities. The useful value of polyherbal combination of medicinal plants will extend beyond the existing understanding of ethnobotanical knowledge for the future researches.

\section{DECLARATIONS}

\section{Acknowledgements}

This project was supported by Atatürk University, Erzurum, Turkey. The authors are very grateful to Mrs. Azam Karimi for her assist in survey, Dr. Saeid Chekani Azar for editing the language, format and grammar of the presented manuscript and also all informants of the study area especially people of Qorveh-e Darjazin districts and Razan town.

\section{Authors' contributions}

Yusuf Kaya: conceptualization, review and editing, supervision, project administration; Zohreh Yousefi: conceptualization, methodology, resources, formal analysis, validation, writing original, review and editing; Mohammad Akrami: review and editing; Mahshad Yousefi: methodology, review, editing, and validation.

\section{Conflict of interest}

The authors declare that there is no conflict of interests.

\section{REFERENCES}

1. Gurib-Fakim A. Medicinal plants: traditions of yesterday and drugs of tomorrow. Molecular Aspects of Medicine. 2006 Feb 1; $27(1): 1-93$. https://doi.org/10.1016/j.mam.2005.07.008

2. Baker DD, Chu M, Oza U, Rajgarhia V. The value of natural products to future pharmaceutical discovery. Natural product reports. 2007; 24(6):1225-44. https://doi.org/10.1039/B602241N

3. Naghibi F, Esmaeili S, Malekmohammadi M, Hassanpour A, Mosaddegh M. Ethnobotanical survey of medicinal plants used traditionally in two villages of Hamedan, Iran.. http://www.rjpharmacognosy.ir/article_5772.html

4. Banerjee M. Public policy on Ayurveda. Economic and Political Weekly, 2002; 37: 1136-1146. https://www.jstor.org/stable/4411901

5. Nezhadali A, Zarrabi-Shirvan B. Separation, Identification and Determination ofVolatile Compounds of Ziziphora persica BungeUsing HS-SPME/GC-MS. International Journal of Environmental Science and Development. 2010 Jun $1 ; 1(2): 115-8$. http://www.ijesd.org/papers/23-B068.pdf

6. Özgen U, Kaya Y, Houghton P. Folk medicines in the villages of Ilıca District (Erzurum, Turkey). Turkish Journal of Biology. 2012 Jan 30; 36(1):93-106.. https://journals.tubitak.gov.tr/biology/abstract.htm?id=12389

7. ICTUR et al. 2005 ed. Trade Unions of the World (6 ${ }^{\text {th }}$ ed.). London, UK: John Harper Publishing. 2005; ISBN 978-0-9543811-5-8.

8. Khorasaninejad S, Mousavi A, Soltanloo H, Hemmati K, Khalighi A. The effect of salinity stress on growth parameters, essential oil yield and constituent of peppermint (Mentha piperita L.). World Applied Sciences Journal. 2010; 11(11):1403-7. Available at https://citeseerx.ist.psu.edu/viewdoc/download?doi=10.1.1.390.9291\&rep=repl\&type=pdf

9. Ghahreman A, Attar F. Biodiversity of plant species in Iran. Central Herbarium of Tehran University; 1999. Google Scholar

10. Jeff-Agboola YA, Awe LB. Antifungal and phytochemical screening of some Nigerian medicinal plant extracts against toxigenic Aspergillus flavus. Cogent Food \& Agriculture. 2016 Dec 31; 2(1):1210556. https://doi.org/10.1080/23311932.2016.1210556

11. Scartezzini P, Speroni E. Review on some plants of Indian traditional medicine with antioxidant activity. Journal of Ethnopharmacology. $2000 \mathrm{Jul}$ 1; 71(1-2):23-43. https://doi.org/10.1016/S0378-8741(00)00213-0

12. Talukdar S, Gupta A. Ethnomedicinal knowledge of the Garo community of two villages in western Assam, India. Journal of Herbal Medicine. 2020; 20:100229. https://doi.org/10.1016/j.hermed.2018.08.004

13. Vitalini S, Iriti M, Puricelli C, Ciuchi D, Segale A, Fico G. Traditional knowledge on medicinal and food plants used in Val San Giacomo (Sondrio, Italy)_An alpine ethnobotanical study. Journal of Ethnopharmacology. 2013 Jan 30;145(2):517-29. https://doi.org/10.1016/j.jep.2012.11.024 
14. Özkan GA, Koyuncu MA. Tradional medicinal plants used in Pınarbaşı area (Kayseri-Turkey). Turkish Journal of Pharmaceutical Sciences. 2005; 2(2):63-82. https://app.trdizin.gov.tr/makale/TlRJeE56UTA/tradional-medicinal-plants-used-in-pinarbasi-area-kayseriturkey-

15. Zare M, Khosravi AR, Joharchi MR. Distribution patterns of the genus Cousinia (Asteraceae) in Iran. Iranian Journal of Botany, $2012 ; 19$ (1): 127-141. https://www.cabdirect.org/cabdirect/abstract/20143317312

16. Ghasemi Pirbalouti A. 2008. Medicinal and Aromatic Plants, (identification and evaluation of their effects). Shahrekord Branch, Islamic Azad University Press. Book, First ed. Iran.

17. Mehriar M, Zaker Haghighi K. Evaluation of the Urban Settlement s Optimal System. International Journal of Architecture and Urban Development. 2012 Oct 1; 2(4):43-50. https://ijaud.srbiau.ac.ir/article_579.html

18. Tahmasebi SH. 2001. Analytical features of the spatial organization of the Hamadan province with an emphasis on city. Hamadan Management and Planning Organization, Hamadan, Iran.

19. Toulmin S and Goodfield J The Ancestry of Science: The Discovery of Time, p. 64, University of Chicago Press (cf. The Contribution of Ibn Sina to the development of Earth sciences Archived 14 March 2010 at the Wayback Machine). 1965. https://scholar.google.com/scholar?cluster $=12146338195224857228 \&$ hl=en\&as $\quad$ sdt $=2005 \& s c i o d t=0,5$

20. McGinnis J Avicenna. Oxford: Oxford University Press. p. 227. 2010; ISBN 978-0-19-533147-9. https://scholar.google.com/scholar?cluster $=16549202591139804681 \&$ hl=en\&as_sdt=2005\&sciodt $=0,5$

21. Zebardast E. City size. Iran Center for Architecture and Urban Studies, Tehran.(in Persian). 2004. https://scholar.google.com/scholar?cluster $=6657000649422093902 \&$ hl=en\&oi=scholarr

22. Rechinger KH. (ed.). Flora Iranica 139b. - Graz, 1980.

23. Meena AK, Bansal P, Kumar S. Plants-herbal wealth as a potential source of ayurvedic drugs. Asian Journal of Traditional Medicines. $2009 \quad$ Aug 20; 4(4):152-70. https://www.researchgate.net/profile/Ajay-Meena-2/publication/266049813 Plantsherbs wealth as a potential source of ayurvedic drugs/links/550262420cf2d60c0e634600/Plants-herbs-wealth-as-a-potentialsource-of-ayurvedic-drugs.pdf

24. Chima UD, Adekunle AT, Okorie MC. Ecological and Ethnomedicinal Survey of Plants within Homesteads in Abia State, Nigeria. Journal of forest and environmental science. 2013; 29(4):257-74. https://doi.org/10.7747/JFS.2013.29.4.257

25. Chan K, Shaw D, Simmonds MS, Leon CJ, Xu Q, Lu A, Sutherland I, Ignatova S, Zhu YP, Verpoorte R, Williamson EM. Good practice in reviewing and publishing studies on herbal medicine, with special emphasis on traditional Chinese medicine and Chinese materia medica. Journal of Ethnopharmacology. 2012 Apr 10; 140(3):469-75. https://doi.org/10.1016/j.jep.2012.01.038

26. Heinrich $M$, Edwards S, Moerman DE, Leonti M. Ethnopharmacological field studies: a critical assessment of their conceptual basis and methods. Journal of Ethnopharmacology. 2009 Jul 6; 124(1):1-7. https://doi.org/10.1016/j.jep.2009.03.043

27. Srivastava S, Lal VK, Pant KK. Polyherbal formulations based on Indian medicinal plants as antidiabetic phytotherapeutics. Phytopharmacology. 2012; 2(1):1-5. http://citeseerx.ist.psu.edu/viewdoc/download?doi=10.1.1.1061.5221\&rep=repl\&type=pdf

28. Mosaddegh M, Naghibi F, Moazzeni H, Pirani A, Esmaeili S. Ethnobotanical survey of herbal remedies traditionally used in Kohghiluyeh va Boyer Ahmad province of Iran. Journal of Ethnopharmacology. 2012 May $7 ; \quad 141(1): 80-95$. https://doi.org/10.1016/j.jep.2012.02.004

29. Nikbakht A, Kafi M. The history of traditional medicine and herbal plants in Iran. InVIII International People-Plant Symposium on Exploring Therapeutic Powers of Flowers, Greenery and Nature 7902004 Jun 4 (pp. 255-258). DOI: https://doi.org/10.17660/ActaHortic.2008.790.37

30. Hardy AD, Walton RI, Vaishnav R. Composition of eye cosmetics (kohls) used in Cairo. International journal of environmental health research. 2004 Feb 1; 14(1):83-91. https://doi.org/10.1080/09603120310001633859

31. Miraldi E, Ferri S, Mostaghimi V. Botanical drugs and preparations in the traditional medicine of West Azerbaijan (Iran). Journal of ethnopharmacology. 2001 May 1; 75(2-3):77-87. https://doi.org/10.1016/S0378-8741(00)00381-0

32. Mirdeilami SZ, Barani H, Mazandarani M, Heshmati GA. 2011. Ethnopharmacological survey of medicinal plants in Maraveh Tappe region, North of Iran. Iranian Journal of Plant Physiology, 2011; 2(1): 325-336. https://eng.iau-saveh.ac.ir/Files/Journal/2012-0402 02.51 .23 6.pdf

33. Madjidi Khameneh F. An ethnomedicine study among women in Uremia (North-West Iran). Collegium antropologicum. $2012 \mathrm{Jul} 10 ;$ 36(2):491-7. https://hrcak.srce.hr/file/126366

34. Reyahi-khoram M, Jafary M, Bayati M, Reyahi-khoram R. Plants and animals diversity in Buqaty Mountain Area (BMA) in Hamadan Province, Iran. Biodiversitas Journal of Biological Diversity. 2012; 13(4). https://doi.org/10.13057/biodiv/d130404

35. Ahvazi M, Khalighi-Sigaroodi F, Charkhchiyan MM, Mojab F, Mozaffarian VA, Zakeri H. Introduction of medicinal plants species with the most traditional usage in Alamut region. Iranian journal of pharmaceutical research: IJPR. 2012; 11(1):185.

36. Safa O, Soltanipoor MA, Rastegar S, Kazemi M, Dehkordi KN, Ghannadi A. An ethnobotanical survey on hormozgan province, Iran. Avicenna journal of phytomedicine. 2013; 3(1):64.

37. Mardaninejad S, Janghorban M, Vazirpour M. Collection and identification of medicinal plants used by the indigenous people of Mobarakeh (Isfahan), southwestern Iran. Journal of Medicinal Herbs,. 2013 Apr 1; 4(1):23-32. http://jhd.iaushk.ac.ir/article 633118.html

38. Mosaddegh M, Esmaeili S, Hassanpour A, Malekmohammadi M, Naghibi F. Ethnobotanical study in the highland of Alvand and $\begin{array}{llllllll}\text { Tuyserkan, Iran. Research Journal of } & 2016 \quad \text { Jan } & \text { 1; } & 3(1): 7-17 .\end{array}$ http://www.rjpharmacognosy.ir/\&url=http:/www.rjpharmacognosy.ir/article_12064_19f6999e9342742606b739bbe2fb7c25.pdf

39. Thomas E, Vandebroek I, Sanca S, Van Damme P. Cultural significance of medicinal plant families and species among Quechua farmers in Apillapampa, Bolivia. Journal of Ethnopharmacology. 2009 Feb 25; 122(1):60-7. https://doi.org/10.1016/j.jep.2008.11.021

40. Fujita T, Sezik E, Tabata M et al. 1995. Traditional medicine in Turkey VII. Econ Bot 49: 406-422.

41. Allchin FR. India: the ancient home of distillation?. Man. 1979 Mar 1:55-63. https://doi.org/10.2307/2801640

42. Sabelli PA, Larkins BA. The development of endosperm in grasses. Plant physiology. 2009 Jan; 149(1):14-26. https://doi.org/10.1104/pp.108.129437

43. De Silva T. Industrial utilization of medicinal plants in developing countries. Medicinal plants for forest conservation and health care. FAO, Rome. 1997:34-44. https://scholar.google.com/scholar?cluster $=7015140001264375935 \& h l=e n \& a s \quad$ sdt=2005\&sciodt=0,5 
44. Krivenko VV, Potebnia GP, Loĭko VV. Experience in treating digestive organ diseases with medicinal plants. Vrachebnoe delo. 1989 Mar l(3):76-8. https://europepmc.org/article/med/2750130

45. Lans C. Comparison of plants used for skin and stomach problems in Trinidad and Tobago with Asian ethnomedicine. Journal of Ethnobiology and Ethnomedicine. 2007 Dec; 3(1):1-2. https://doi.org/10.1186/1746-4269-3-3

46. Kim $\mathrm{H}$, Song MJ. Traditional plant-based therapies for respiratory diseases found in North Jeolla Province, Korea. The Journal of Alternative and Complementary Medicine. 2012 Mar 1; 18(3):287-93. https://doi.org/10.1089/acm.2010.0848

47. Adhami HR, Farsam H, Krenn L. Screening of medicinal plants from Iranian traditional medicine for acetylcholinesterase inhibition. Phytotherapy Research. 2011 Aug; 25(8):1148-52. https://doi.org/10.1002/ptr.3409

48. Kim H, Song MJ, Brian H, Choi K. A comparative analysis of ethnomedicinal practices for treating gastrointestinal disorders used by communities living in three national parks (Korea). Evidence-Based Complementary and Alternative Medicine. 2014 Jan 1; 2014. https://doi.org/10.1155/2014/108037

49. Shyamal S, Latha PG, Shine VJ, Suja SR, Rajasekharan S, Devi TG. Hepatoprotective effects of Pittosporum neelgherrense Wight\&Arn., a popular Indian ethnomedicine. Journal of ethnopharmacology. 2006 Aug 11; 107(1):151-5. https://doi.org/10.1016/j.jep.2006.02.018

50. Sharma J, Gairola S, Gaur RD, Painuli RM. The treatment of jaundice with medicinal plants in indigenous communities of the SubHimalayan region of Uttarakhand, India. Journal of Ethnopharmacology. 2012 Aug 30; 143(1):262-91. https://doi.org/10.1016/j.jep.2012.06.034

51. Hossan S, Agarwala B, Sarwar S, Karim M, Jahan R, Rahmatullah M. Traditional use of medicinal plants in Bangladesh to treat urinary tract infections and sexually transmitted diseases. Ethnobotany Research and Applications. 2010 Apr 7; 8:061-74. https://ethnobotanyjournal.org/index.php/era/article/view/304

52. Sharafzadeh S, Alizadeh O. Some medicinal plants cultivated in Iran. Journal of Applied Pharmaceutical Science. 2012; 2(1):134-7. http://japsonline.com/admin/php/uploads/358 pdf.pdf

53. Afifi-Yazar FU, Kasabri V, Abu-Dahab R. Medicinal plants from Jordan in the treatment of diabetes: traditional uses vs. in vitro and in vivo evaluations-part 2. Planta medica. 2011 Jul; 77(11):1210-20. DOI: https://doi.org/10.1055/s-0031-1279983

54. Fabrega H. An ethnomedical perspective of Anglo-American psychiatry. The American journal of psychiatry. 1989 May. https://doi.org/10.1176/ajp.146.5.588

55. Kim H, Song MJ. Oral traditional plant-based therapeutic applications for pain relief recorded in North Jeolla province, Korea. http://nopr.niscair.res.in/handle/123456789/22192

56. Abdillah S, Tambunan RM, Sinaga YM, Farida Y. Ethno-botanical survey of plants used in the traditional treatment of malaria in Sei Kepayang, Asahan of North Sumatera. Asian Pacific journal of tropical medicine. 2014 Sep 1; 7: S104-7. https://doi.org/10.1016/S19957645(14)60213-3

57. Parsa A. Medicinal plants and drugs of plant origin in Iran. I. Qualitas Plantarum et Materiae Vegetabiles. 1959 Mar 1;5(4):375-94. https://link.springer.com/content/pdf/10.1007/BF01099755.pdf

58. Wu R, Wang L, Kuo HC, Shannar A, Peter R, Chou PJ, Li S, Hudlikar R, Liu X, Liu Z, Poiani GJ. An update on current therapeutic drugs treating COVID-19. Current pharmacology reports. $2020 \mathrm{Jun} ; 6(3): 56-70$. https://doi.org/10.1007/s40495-020-00216-7

59. Mitjà O, Clotet B. Use of antiviral drugs to reduce COVID-19 transmission. The Lancet Global Health. 2020 May 1;8(5):e639-40. https://doi.org/10.1016/S2214-109X(20)30114-5

6o. Perrone F, Piccirillo MC, Ascierto PA, Salvarani C, Parrella R, Marata AM, Popoli P, Ferraris L, Marrocco-Trischitta MM, Ripamonti D, Binda F. Tocilizumab for patients with COVID-19 pneumonia. The single-arm TOCIVID-19 prospective trial. Journal of translational medicine. 2020 Dec; 18(1):1-1. https://doi.org/10.1186/s12967-020-02573-9

61. Brown AJ, Won JJ, Graham RL, Dinnon III KH, Sims AC, Feng JY, Cihlar T, Denison MR, Baric RS, Sheahan TP. Broad spectrum antiviral remdesivir inhibits human endemic and zoonotic deltacoronaviruses with a highly divergent RNA dependent RNA polymerase. Antiviral research. 2019 Sep 1; 169:104541. https://doi.org/10.1016/j.antiviral.2019.104541

62. Baden LR, Rubin EJ. Covid-19 — the search for effective therapy. https://doi.org/10.1056/NEJMe2005477

63. Zhao JP, Hu Y, Du RH, Chen ZS, Jin Y, Zhou M, Zhang J, Qu JM, Cao B. Expert consensus on the use of corticosteroid in patients with 2019-nCoV pneumonia. Zhonghua jie he he hu xi za zhi= Zhonghua jiehe he huxi zazhi= Chinese journal of tuberculosis and respiratory diseases. 2020 Mar 1; 43(3):183-4. DOI: https://doi.org/10.3760/cma.j.issn.1001-0939.2020.03.008

64. US Food and Drug Administration. CytoSorb ${ }^{\circledR} 300 \mathrm{~mL}$ Device Approved by FDA for Emergency Treatment of COVID-19. N (\%) or median (range). 2020. https://www.fda.gov/media/136866/download

65. Bernal JL, Andrews N, Gower C, Gallagher E, Simmons R, Thelwall S, Tessier E, Groves N, Dabrera G, Myers R, Campbell C. Effectiveness of COVID-19 vaccines against the B. 1.617. 2 variant. medRxiv. 2021 Jan 1. https://doi.org/10.1101/2021.05.22.21257658

66. Mrityunjaya M, Pavithra V, Neelam R, Janhavi P, Halami PM, Ravindra PV. Immune-boosting, antioxidant and anti-inflammatory food supplements targeting pathogenesis of COVID-19. Frontiers in Immunology. 2020; 11. https://doi.org/10.3389/fimmu.2020.570122

67. Soto ME, Guarner-Lans V, Soria-Castro E, Manzano Pech L, Pérez-Torres I. Is antioxidant therapy a useful complementary measure for Covid-19 treatment? An algorithm for its application. Medicina. 2020 Aug; 56(8):386. https://doi.org/10.3390/medicina56080386 